\title{
1 Decoding neuronal diversity by single-cell Convert-seq
}

2 Joachim Luginbühl'1,2, Tsukasa Kouno ${ }^{1,2}$, Rei Nakano1,3,4, Thomas E Chater ${ }^{5}$, Divya M Sivaraman ${ }^{1,2,6}$,

3 Mami Kishima ${ }^{1,3}$, Filip Roudnicky ${ }^{7}$, Piero Carninci ${ }^{1,8}$, Charles Plessy ${ }^{1,8}$, Jay W Shin ${ }^{1,2,9, *}$

$5 \quad{ }^{1}$ RIKEN Center for Life Science Technologies, Division of Genomic Technologies, RIKEN Yokohama, 230-0045, 6 Japan; ${ }^{2}$ RIKEN Center for Integrative Medical Sciences, Laboratory for Advanced Genomic Circuits, RIKEN

7 Yokohama, 230-0045, Japan; ${ }^{3}$ RIKEN Center for Integrative Medical Sciences, Laboratory for Cellular Function

8 Conversion Technology, RIKEN Yokohama, 230-0045, Japan; ${ }^{4}$ Nihon University College of Bioresource Sciences,

9 Department of Veterinary Medicine, Laboratory of Veterinary Biochemistry, 252-0880, Japan; ${ }^{5}$ RIKEN Center for

10 Brain Science, Laboratory for Synaptic Plasticity and Connectivity, RIKEN Wako, 351-0198, Japan; ${ }^{6}$ Sree Chitra

11 Tirunal Institute for Medical Sciences and Technology, Thiruvananthapuram, Kerala, 695011, India; ${ }^{7}$ Institute of

12 Pharmaceutical Sciences, ETH Zurich, Zurich, Switzerland; ${ }^{8}$ RIKEN Center for Integrative Medical Sciences,

13 Laboratory for Single Cell Technologies, RIKEN Yokohama, 230-0045, Japan; 'Lead contact

*Materials and correspondence: Jay.Shin@riken.jp

\section{Summary}

The conversion of cell fates is controlled by hierarchical gene regulatory networks (GRNs) that induce remarkable alterations in cellular and transcriptome states. The identification of key regulators within these networks from myriad of candidate genes, however, poses a major research challenge. Here we present Convert-seq, combining single-cell RNA sequencing (scRNA-seq) and pooled ectopic gene expression with a new strategy to discriminate sequencing reads derived from exogenous and endogenous transcripts. We demonstrate Convert-seq by associating hundreds of single cells during reprogramming of human fibroblasts to induced neurons (iN) with exogenous and endogenous transcriptional signatures. Convert-seq identified GRNs modulating the emergence of developmental trajectories and predicted combinatorial activation of exogenous transcription factors controlling iN subtype specification. Functional validation of iN subtypes generated by novel combinations of exogenous transcription factors establish Convert-seq as a broadly applicable workflow to rapidly identify key transcription factors and GRNs orchestrating the reprogramming of virtually any cell type. 


\section{Introduction}

33 Fully differentiated cells can be reprogrammed to alternative fates via ectopic expression of defined 34 combinations of transcription factors and/or microRNAs (Chanda et al., 2014; Hu et al., 2015; Mertens 35 et al., 2015; Pang et al., 2011; Pfisterer et al., 2011; Ryoji Amamoto and Arlotta, 2014; Victor et al., 36 2014; Vierbuchen et al., 2010; Xue et al., 2013). Reprogrammed cells offer invaluable alternative routes

37 for the treatment of various diseases and represent a substantial improvement to the field of disease 38 modeling (Masserdotti et al., 2016). However, despite the establishment of new in silico prediction tools 39 (Rackham et al., 2016), it has remained a challenge to identify essential genetic drivers that determine 40 specific subtypes of cells during reprogramming.

41 Cell reprogramming is of particular interest for human neurons, which are notoriously difficult 42 to obtain from patients and cannot be expanded in culture (Ryoji Amamoto and Arlotta, 2014). Since 43 the first report on reprogramming of induced neurons (iN), several studies have succeeded in identifying 44 defined combinations of neurogenic transcription factors that allow the reprogramming of specific neuronal subtypes (Masserdotti et al., 2016). For instance, distinct sets of neurogenic transcription factors have been shown to create dopaminergic neurons and cholinergic motor neurons that are selective targets of degeneration in patients with Parkinson's disease and amyotrophic lateral sclerosis (ALS), respectively (Caiazzo et al., 2011; Jiang et al., 2015; Kim et al., 2011; Liu et al., 2013, 2016; Mazzoni et al., 2013; Pfisterer et al., 2011; Son et al., 2011). However, an inherent limitation of studies aiming to identify key transcription factors controlling cell reprogramming is that the identification of candidates from myriad of transcription factors still is based on trial and error. This is exacerbated by the time and labor intensive retesting and validation of newly identified candidates. Pooled screens, instead of one-by-one tests, offer a more efficient and scalable strategy, but they either rely on simple phenotypic readouts or require specific markers to enrich for target cell populations, which are often not available (Chen et al., 2015; Hsu et al., 2014; Liu et al., 2018; Shalem et al., 2015). This is particularly problematic with regard to reprogramming, which is intrinsically inefficient, resulting in heterogenous cell populations containing unreprogrammed, partially reprogrammed, and successfully reprogrammed cells.

To address this challenge, we developed Convert-seq, combining ectopic expression of transcription factors with single-cell RNA sequencing (scRNA-seq) to retrospectively associate single cells with their exogenous and endogenous expression profiles. Convert-seq represents a pooled 
62 (multiplexed) screening strategy that allows to identify transcription factors and GRNs governing the 63 reprogramming of many different celltypes in a single experiment. We demonstrate Convert-seq in iN 64 generated by the pooled infection of human fibroblasts with 20 pro-neuronal transcription factors using 65 two single-cell sequencing platforms, the Fluidigm $\mathrm{C} 1$ system based on microfluidic technology and the 66 10x Genomics system based on nano-droplets. By association of different transcriptional states and 67 developmental trajectories emerging during reprogramming with distinct exogenous and endogenous transcriptional signatures, we identified defined combinations of key transcription factors orchestrating the reprogramming of human fibroblasts into multiple neuronal subtypes. Our data demonstrate the ability of Convert-seq to efficiently gain rich insight into biological processes governing cell plasticity and cell fate decisions and to systematically dissect GRNs controlling subtype specification during cell reprogramming.

\section{Results}

\section{Generation of a heterogenous population of human induced neurons}

77 To enable systematic identification of combinations of transcription factors governing the reprogramming of human fibroblasts into specific neuronal subtypes, we generated a pool of lentiviruses (hereafter termed transcription factor-pool or TF-pool) encoding 20 pro-neuronal transcription factors. We included the pioneer factor ASCL1 (Wapinski et al., 2013) and several transcription factors that increase the efficiency of neuronal reprogramming (POU3F2, ZIC1, OLIG2, NEUROD1) (Pang et al., 2011; Vierbuchen et al., 2010). To promote reprogramming of different subtypes of neurons, we further included transcription factors which have been previously shown to convert human fibroblasts into GABAergic (DLX1, DLX2) (Victor et al., 2014), cholinergic (NEUROG2, ISL1) (Liu et al., 2013; Son et al., 2011), serotonergic (FEV) (Xu et al., 2016) or dopaminergic (FOXA2, NR4A2, PITX3) (Liu et al., 2012; Pfisterer et al., 2011) neurons when co-expressed with other transcription factors. Additionally, we selected 7 transcription factors which have not yet been used to generate specific neuronal subtypes, but showed significant up-regulation during the differentiation of human induced pluripotent stem cells (iPSC) into neuronal progenitor cells (NPC) (Figure S1A). Each transcription factor was expressed under the elongation factor 1 alpha (EF1A) promoter together with a gene encoding Venus 
92 the multiplicity of infection (MOI) of each lentivirus to allow $\sim 85 \%$ of fibroblasts to express mixed 93 combinations of 2 - 6 transcription factors after stochastic infection with the TF-pool (Figure 1A and 94 Figure S1C). Using successive application of neuronal induction medium for 14 days followed by 95 neuronal maturation medium for 7 days, we successfully transformed fibroblasts into cells exhibiting hetergeneous neuronal morphologies and expressing canonical neuronal marker genes (Figures 1B). At 9 days post-infection (dpi), $78.6 \%$ of fibroblasts (hereafter termed transcription factor-induced cells or TFi) stained positive for the immature neuronal marker TUBB3 and 34.7\% expressed MAP2, a microtubule-associated protein expressed specifically in neurons (Jeff et al., 1988) (Figure 1C). By 21 dpi, TUBB3+ and MAP2+ ratios increased to $93.5 \%$ and $42.4 \%$, respectively, indicating progressive differentiation towards the neuronal lineage. Consistent with the adoption of a neuronal fate, quantitative polymerase chain reaction ( $\mathrm{PPCR}$ ) revealed gradual upregulation of pan-neuronal marker genes (MAP2, NRCAM, NEUN, SYN1) and established neuronal subtype marker genes (SLC17A7 [glutamatergic neurons], GABRA1 [GABAergic neurons], $T H$ [dopaminergic neurons] and CHAT [cholinergic neurons]) as well as gradual downregulation of fibroblast marker gene expression (VIM, SNAl1) in TFi starting at $7 \mathrm{dpi}$ (Figures 1D). induced neurons (iN) solely by chemical cocktails of small molecules (Hu et al., 2015; Li et al., 2015). Thus, to discriminate between transcription factor-mediated and chemical-mediated conversion, we separately cultured fibroblasts transduced with lentiviruses expressing YFP only (hereafter termed chemically induced cells or $\mathrm{Ci}$ ) using the same neuronal induction regime. Interestingly, we found that similar to TFi, application of small molecules and growth factors alone induced the development of TUBB3+ neurites, upregulated the expression of NEUN, SYN1, SLC17A7 and TUBB3 and downregulated the expression of VIM and SNAI1 in Ci (Figures 1B-1D). However, Ci failed to express MAP2 at any time-point analyzed, indicating that the small molecules we used were insufficient to elicit neuronal maturation on their own. Consistently, neuronal complexity, measured as number of branch

117 points and average neurite length, was significantly increased at $9 \mathrm{dpi}$ (8.5-fold and 1.8-fold, 118 respectively) and at $21 \mathrm{dpi}$ (5.6-fold and 4.8-fold, respectively) when fibroblasts were infected with the 119 TF-pool compared to application of small molecules alone (Figures S1E-S1F). 
122 Immunofluorescence for canonical neuronal subtype markers at 21 dpi revealed that TFi differentiated 123 into a heterogeneous population of cells expressing glutamatergic (vGLUT1+; 29\%), GABAergic 124 (GABA+; 23\%), cholinergic (CHAT+; 19\%) and dopaminergic ( $\mathrm{TH}+; \sim 4 \%)$ subtype-specific genes 125 (Figures 1E-1F). In contrast, only $\sim 15 \%$ of Ci expressed vGLUT1 at $21 \mathrm{dpi}$ and none of the other 126 subtype-specific genes was induced by small molecules.

To gain further insight into the molecular heterogeneity of TFi and $\mathrm{Ci}$, we used droplet-based 128 massively parallel scRNA-seq (Zheng et al., 2017) to profile 2,092 $\mathrm{Ci}$ and 1,900 TFi at $14 \mathrm{dpi}$ (Table 129 S1). Batch-corrected clustering of all cells that passed quality control using $t$-Distributed Stochastic 130 Neighbor embedding ( $t$-SNE) separated $\mathrm{Ci}$ and TFi along $t$-SNE 2 (Figures 2A and S2A-D). Correlation 131 analysis of top genes along $t$-SNE 2 (Pearson correlation; $p<10^{-7}$ ) revealed activation of genes relevant 132 for neuronal differentiation (NRCAM, STMN2, SST, DKK3) and synapse formation (SYT1, SERPINI1, 133 SYNGR1, SYNPO2), which was accompanied by a decrease in fibroblast-specific genes (SNAI2, 134 THY1) (Figure 2B). Other suppressed genes included extracellular matrix genes (COL3A1, COL15A1, $135 E D I L 3, H A P L N 1, C P X M 1$ and MFAP4), reflective of the morphological changes that occur during the conversion of iN. t-SNE further partitioned TFi cells into one main cluster (CL3) and several 137 transcriptionally distinct clusters (CL4-CL9). CL5 dominantly expressed cell cycle-related genes including MIKI67 and TOP2A and showed enrichment of Gene ontology (GO) terms related to cell division, indicating that these cells - possibly un-reprogrammed fibroblasts - remained in a mitotic stage

140 (Figures 2C-D; Table S2). Importantly, consistent with morphology and marker expression stainings, clusters CL4 and CL6 - CL9 all showed enrichment in terms related to nervous system development and neurogenesis. To annotate clusters CL4 - CL9, we interrogated top differentially expressed genes 143 (Seurat; $p<10^{-20}$ ) for known neuronal subtype marker genes. This revealed CL4 to associate with 144 immature neurons, CL6 with the motor neuron program (CHRNA5, CP), CL7 with the GABAergic 145 neuron program (ANK3, NKAIN4), CL8 with the glutamatergic neuron program (SLC6A17, INSM1) and 146 CL9 with the dopaminergic neuron program (DRD4, SEMA3G) (Figures 2E-F and S2E).

147 Together, these data revealed that transduction of our TF-pool converted fibroblasts into a 148 population of cells exhibiting a high degree of heterogeneity and distinct neuronal subtype-specific 149 molecular signatures. 
152 To decode the identity of exogenous transcription factors in individual cells, we designed Convert-seq,

153 combining the stochastic nature of pooled, virus-based screens with massively parallel scRNA-seq

154 (Figure 1A). Based on full-length reads, we extracted nucleotides at the $5^{\text {' }}$ and 3 ' junctions of exogenous

155 ORFs and assembled artificial transcript models of endogenous and exogenous reference sequences

156 (Figure 3A). Alignment of reads to these specific 5' and 3' junctions allows to discriminate between

157 exogenous and endogenous gene expression. To benchmark the accuracy and sensitivity of Convert-

158 seq, we profiled gene expression of bulk fibroblasts infected with single transcription factors, two 159 combinations of 10 transcription factors, and the complete TF-pool at two multiplicity of infections (MOI)

160 (Figures 3B and S3A). We also included chemically-induced ( $\mathrm{Ci}$ ) cells and uninfected fibroblasts as 161 controls. Sequence alignment to the artificial transcript model using Bowtie (Langmead et al., 2009) 162 revealed specific detection of exogenous transcription factors, but resulted in $24.4 \%$ false-negative 163 events, possibly due to the exclusion of multi-mapping reads (Figure 3C). To reduce the number of 164 false-negative events, we implemented the pseudo-alignment tool Kallisto, providing the advantage of 165 assigning multi-mapping reads to transcripts without pinpointing exactly how the sequences of the reads 166 and transcripts align (Bray et al., 2016). Pseudoalignment with Kallisto reduced the occurrence of false 167 negative events to $6.3 \%$ and yielded highly specific and sensitive detection efficiencies of $97.5 \%$ and $87.5 \%$ in individual infection and pooled infection samples, respectively (Figures 3D and S3B).

To further confirm specific detection of lentivirus-mediated expression, we revealed significantly higher mean expression of exogenous transcription factors compared to the mean expression of corresponding endogenous transcription factors $(p<0.05$; Figure $3 E)$. Interestingly, additional infection experiments revealed that for several genes, ectopic expression failed to activate endogenous expression, while we observed upregulation of endogenous $D L X 1, D L X 2$, ISL1, NEUROD1, PAX6 and

174 ZIC1 transcripts after infection with their exogenous counterparts (Figure S3C).

Induced neurons resemble primary human newborn neurons in global gene

178 Convert-seq requires full-length coverage of transcripts to obtain sequencing reads falling into specific 1795 ' and 3' junction sequences. Accordingly, detection of exogenous genes from the 3'-biased dropletbased ScRNA-seq data was inefficient (Figure S3D). Therefore, we sequenced transcription factor- 
181 induced cells (TFi) and chemically induced cells ( $\mathrm{Ci})$ at an early ( $9 \mathrm{dpi})$ and late ( $21 \mathrm{dpi})$ time point (216 cells and 152 cells, respectively) on the Fluidigm C1 platform using Smartseq2 technology (Figure 4A).

183 Unsupervised clustering of all cells that passed quality control using $t$-SNE identified 5 transcriptionally distinct clusters of cells that separated largely by type rather than batch (Figures 4B and S4A-F). GO analysis of differentially expressed genes between all clusters (fold-change $>2 ; p<10^{-4}$ ) revealed that CL1 dominant in unreprogrammed fibroblasts specifically expressed genes related to cell cycle and cell division, whereas CL2 dominant in $\mathrm{Ci}$ at $9 \mathrm{dpi}$ was characterized by specific expression of genes related to general developmental processes without bias towards nervous system development (Figures $4 \mathrm{C}$ and S4G-H; Table S3). CL3 dominant in TFi at 9 dpi expressed genes related to the inflammatory response and cell cycle regulation, indicating inefficient initiation of the reprogramming process. Similar to CL2, CL4 dominant in TFi at $21 \mathrm{dpi}$ expressed genes related to general developmental processes.

192 Finally, CL5 expressed genes related to neuron development and neuron maturation, suggesting that CL5 contained cells that were most efficiently reprogrammed towards the neuronal lineage. scRNA-seq dataset of human primary cortical and medial ganglionic eminence samples to infer lineage relationships among the cells in an adjacency network on the basis of pairwise correlations between cells (Nowakowski et al., 2017) (Figure 4D). This analysis revealed that while Ci correlated with alternative cell types including endothelial cells and mural cells, TFi showed progressive correlation with newborn neurons, excitatory neurons and inhibitory neurons. Consistent with our previous results

200 (Figure S4G-H), we found that TFi exhibiting transcriptional profiles related to cell cycle regulation and inflammatory response showed stronger correlation with neuronal progenitors and newborn neurons, whereas the majority of TFi exhibiting transcriptional profiles related to neuron development and neuron maturation correlated with excitatory and inhibitory neurons (Figure 4E).

204

\section{Association of developmental trajectories with exogenous expression profiles}

206 A previous study in mouse embryonic fibroblasts showed that reprogramming to neurons occurs

207 through a continuum of intermediate states, whereby ectopic expression of specific exogenous transcription factors is required to suppress aberrant developmental trajectories and maintain neuronal

209 identity (Treutlein et al., 2016). To analyze intermediate states in our time-course data and simultaneously associate the expression of exogenous transcription factors with distinct developmental 
211 trajectories during reprogramming, we first used single-cell Convert-seq to assign individual cells with

212 exogenous transcription factors and corresponding endogenous gene expression profiles (Figure 4F).

213 Next, we used the Monocle algorithm (Trapnell et al., 2014) to place the cells in pseudo-temporal order

214 based on differentially expressed genes between $9 \mathrm{dpi}$ and $21 \mathrm{dpi}$ (qval < 0.01) (Figure S5A). This

215 revealed that cells bifurcated into two divergent developmental trajectories shortly after initiation of

216 reprogramming. Using branch-specific differential gene expression (fold-change $>4 ; p<10^{-2}$ ), we found

217 that branch 1 cells contained mostly TFi and Ci from 9 dpi and expressed genes involved in viral

218 expression, ribosome biogenesis and cell cycle control, whereas branch 2 cells contained TFi and $\mathrm{Ci}$

219 from both $9 \mathrm{dpi}$ and $21 \mathrm{dpi}$, and expressed genes related to nervous system development (Figures S5B-

220 C; Table S4). Next, we correlated differentially expressed genes across pseudotime. Unexpectedly,

221 positively correlating genes $(r>0.2)$ were enriched for $G O$ terms related to various developmental

222 processes (Figures S5D-E, Table S5), implying the emergence of distinct developmental trajectories

223 during reprogramming. Indeed, assessment of gene expression levels along pseudotime confirmed that

224 neurogenic genes (NRCAM, SFRP1, SNAP25 and SYT1) and genes regulating the development of

225 mesodermal tissues including bone (BMP4), kidney (FAT4) and endothelial cells (PGF and VEGFA)

226 were consistently up-regulated and followed a similar expression trajectory pattern during the

227 reprogramming process (Figure S5F-H).

228 To identify exogenous transcription factors governing the emergence of distinct developmental

229 trajectories, we reordered the cells using a set of 3,322 genes previously implicated in various

230 developmental processes (Figure 5A; Table S6). This revealed bifurcation of cells into two main

231 trajectories, separating the majority of $\mathrm{Ci}$ and part of early TFi (branch 1) from the rest of TFi (branch

232 2). Branch-specific $G O$ analysis (fold-change $>4 ; p<0.05$ ) suggested association of branch 2 with cell

233 fate commitment towards the neuronal lineage, while branch 1 was associated with alternative

234 developmental fates (Figures 5B-C; Table S7). Notably, no exogenous transcription factor was

235 specifically enriched in branch 1 (Fisher's exact test; $p>0.05$ ) (Figures 5D and S5I). In contrast, branch

2362 showed significant enrichment of 10 exogenous transcription factors (ASCL1, DLX1, DLX2, FEV,

237 FOXP2, ISL1, NEUROG2, NR4A2, PAX6 and ZIC1; $p<10^{-2}$ ). We independently transduced fibroblasts

238 with the complete TF-pool (20 transcription factors), the 10 transcription factors enriched in branch 1 ,

239 and the 10 transcription factors showing no enrichment in either branch. Neuronal profiling using

240 immunofluorescence for TUBB3 and MAP2 and qPCR for pan-neuronal, neurotransmitter and fibroblast 
241 marker genes revealed that infection with transcription factors enriched in branch 2 markedly increased

242 the efficiency of neuronal reprogramming compared to both the complete TF-pool and transcription

243 factors that showed no branch-specific enrichment (Figures 5E-H). In fact, infection with transcription

244 factors without branch-specific enrichment reduced the efficiency of neuronal conversion compared to

245 the complete TF-pool and upregulated genes that were enriched in branch 1, such as AREG and

246 PTHR1, during pseudo-temporal ordering (Figure 5I). Collectively, these data revealed that the cocktail

247 of small molecules converted cells into „uncommited“ cells, whereas combination of small molecules

248 with ectopic expression of defined transcription factors enhanced the conversion towards the neuronal

249 lineage.

250

251 Identification of GRNs controlling subtype specification of induced neurons

252 In previous reports, a single transcription factor was sufficient to reprogram cells to iN (Chanda et al., 253 2014; Liu et al., 2013). The reprogramming of the vast majority of neuronal subtypes, however, requires 254 combinatorial expression of transcription factors in the same cell (Tsunemoto et al., 2018). To infer 255 combinations of exogenous transcription factors governing neuronal subtype specification from our 256 single-cell data, we classified each subtype based on canonical marker genes for glutamatergic, 257 cholinergic, dopaminergic, GABAergic, serotonergic, glycinergic neurons, and neuronal progenitor cells 258 (NPC). Next, exogenous transcription factors were assigned to each neuronal subtype network based 259 on significant association with at least 3 endogenous marker genes (Fisher's exact tests; $p<0.05$ ) 260 (Figure 6A). For NPC and glycinergic subtypes, only a single exogenous transcription factor (ISL1 and 261 PAX6, respectively) showed 3 or more associations, while no exogenous transcription factor showed more than 2 associations with serotonergic genes (Figure S6A). Notably, however, we identified various combinatorial enrichements of exogenous transcription factors associated with three or more of glutamatergic-, cholinergic-, dopaminergic- and GABAergic-related genes. Previous studies have shown that combination of $D L X 1, D L X 2$ and CTIP2 with miR-9/9*-124 converted human fibroblasts into an enriched population of neurons analogous to GABAergic striatal medium spiny neurons, whereas combination of FOXA2, NR4A2 and PITX3 with additional transcription factors generated dopaminergic neurons (Caiazzo et al., 2011; Liu et al., 2012; Pfisterer et al., 2011; Victor et al., 2014). Our results, in part, are consistent with these studies; two of the four transcription factors associating with the 
271 associating with the dopaminergic GRN were FOXA2, NR4A2 and PITX3 (Figure 6B). Moreover, ISL1,

272 which was previously combined with other transcription factors to convert human fibroblasts into 273 cholinergic motor neurons (Son et al., 2011), associated stronger to the cholinergic GRN than to other 274 GRNs. However, the distribution of transcription factors among subtype-specific GRNs was not mutually 275 exclusive. For example, the glutamatergic GRN comprised transcription factors of all other GRNs, but 276 further included ASCL1, FEV, NEUROG2. Thus, our data support a hierarchical reprogramming model 277 which predicts that replacement of only a few factors can alter the fate of generated cells (Wapinski et 278 al., 2013), and suggests that few transcription factors can override other transcription factors when co279 expressed in the same cell.

280 Subsequently, we independently tested all four predicted neuronal subtype combinations and 281 evaluated expression of VGLUT1, CHAT, TH and GABA in reprogrammed neurons (Figures 6C and 282 S6B). In all combinations analyzed, ectopic expression of predicted combinations significantly enriched 283 for the intended target subset when compared to the complete TF-pool control $(p<0.01)$.

284 To identify minimal and robust combinations of transcription factors in glutamatergic and cholinergic GRNs, we attributed all genes Combination Scores (CS; Supplementary Methods). The CS represents the significance of cumulative marker gene expression in cells containing at least 2 of the predicted exogenous transcription factors (Figures 6D-E). In a second line of validation, we chose the gene with the highest CS for each network (GRIK2 for the glutamatergic network and CHRNA1 for the cholinergic network) and infected fibroblasts with predicted combinations of exogenous transcription factors. We evaluated phenotypes of iN at 21 dpi using immunofluorescence for VGLUT1, CHAT, TH and GABA. Infection with GRIK2-associated (DLX2, NEUROG2, PAX6, ZIC1) and CHRNA1-associated

292 (DLX1, ISL1, NEUROG2, PAX6) transcription factors significantly enriched for glutamatergic and 293 cholinergic neurons, respectively, when compared to fibroblasts infected with the complete TF-pool and 294 Ci. Notably, PAX6, which associated with all four neuronal subtype-specific GRNs (Figures 6A-B), also 295 was included in each of the newly identified combinations, indicating that similar to its role during normal neuronal development (Stoykova et al., 2000; Yun et al., 2001), PAX6 acts as a master regulator during neuronal reprogramming. characterized the electrophysiological properties of iN infected with DLX2, NEUROG2, PAX6, ZIC1 
301 combinations of transcription factors could generate repetitive action potentials upon current injection

302 (Figure 6F-G). We confirmed that these action potentials were inhibited by withdrawal of extracellular

$303 \mathrm{Na}^{+}$. Notably, we observed that glutamatergic and cholinergic iN fired action potentials exhibiting

304 different wave forms and showed significant differences in resting membrane potential and action

305 potential duration, suggesting that the infection of the two different combinations of transcription factors

306 generated iN with distinct electrophysiological properties.

307 Collectively, these results demonstrate that Convert-seq enabled efficient identification of GRNs and

308 defined transcription factors directing the reprogramming of fibroblasts towards distinct, functional 309 neuronal subtypes.

310

\section{Discussion}

312 We developed Convert-seq, a method based on a previously published nested-PCR-based single-cell 313 screening system (Shin et al., 2012), which adapts retrospective identification of vector-based gene expression in single-cells for the scale of massively-parallel single-cell RNA sequencing. Convert-seq

315 overcomes the laborious and time-consuming process of identifying specific combinations of transcription factors capable to reprogram the original transcriptional network(Vierbuchen and Wernig,

317 2011). Particularly in less well-characterized cell types, it is a daunting task to test all possible 318 combinations of candidate reprogramming factors in a one-by-one approach. The intricacy to 319 disentangle the roles of exogenous and endogenous genes during reprogramming further complicates 320 matters, which is why, despite considerable success in the reprogramming of various cell types, still 321 little is known about gene regulatory networks (GRNs) controlling the reprogramming process(Gascon 322 et al., 2017).

323 We have demonstrated that when combined with SMART-seq technology (Picelli et al., 2014;

324 Ramskold et al., 2012), Convert-seq can reliably discriminate between endogenous and exogenous 325 transcripts both at the bulk and single-cell level. Thus, this method allows to analyze transcriptional 326 effects initiated by different combinations of candidate reprogramming factors in a single experiment. 327 Since the number of single cells required to reach statistical significance grows exponentially as the 328 number of candidate genes increases, Convert-seq is incapable of profiling all possible combinations. 329 Therefore, we have limited our study to a subset of possible combinations by adjusting the multiplicity 330 of infection (MOI) and culture conditions that favour the survival of neuronal cells (i.e. positive selection). 
331 Implementing Convert-seq in higher throughput platforms such as droplet-based scRNA-seq will further 332 strengthen the utility of this approach. We tested Convert-seq in a droplet based 3'-seq platform, 333 however, detection of exogenous transcripts was inefficient, possibly due to the strong 3 ' bias of the protocol. Future work could provide droplet-based scRNA-seq platforms with more even coverage across transcripts or encode the identity of exogenous genes in sequence barcodes at the $3^{\text {' }}$-end of constructs (Adamson et al., 2016; Dixit et al., 2016; Jaitin et al., 2016). field of reprogramming. However, using Convert-seq, we identified distinct developmental trajectories emerging early during the reprogramming of human fibroblasts to induced neurons and identified a set of 10 exogenous transcription factors to repress alternative developmental programs. Moreover, by

341 discriminating exogenous and endogenous genes in single-cells, Convert-seq identified distinct 342 combinations of transcription factors controlling the reprogramming of fibroblasts into different neuronal 343 subtypes exhibiting distinct electrophysiological properties.

In addition to providing alternative sources for cell-based therapies, cell reprogramming could help to decipher the molecular logic behind subtype specification during normal development. Strikingly, our data recapitulates some of the general principles of normal neuronal development. For example, PAX6, a paired box transcription factor that acts as master regulator of the mammalian nervous system development and is expressed in region-specific manner in neuronal progenitor cells (NPCs) and uniformely in neuroectodermal cells differentiated from embryonic stem cells (ESCs) and iPSCs 350 (Chapouton et al., 1999; Stoykova et al., 2000; Yun et al., 2001; Zhang et al., 2010), associated with all neuronal subtypes. PAX6-expressing neuroectodermal cells can be readily patterned to region specific NPCs that give rise to various neuronal subtypes including cholinergic neurons ( $\mathrm{Li}$ et al., 2005), dopaminergic neurons (Yan et al., 2005) and GABAergic neurons (Kallur et al., 2008). Thus, our findings

354 indicate that PAX6 acts as a master regulator during reprogramming of iNs that, when combined with 355 additional transcription factors, can instruct the specification into diverse neuronal subtypes. In line with 356 this, our results show that combination of PAX6 with NEUROG2, DLX2 and ZIC1 generates mainly 357 glutamatergic neurons, whereas substitution of $D L X 2$ and ZIC1 with $D L X 1$ and ISL1 generates mainly cholinergic neurons. These findings are partly consistent with previous studies; in the dorsal telencephalon, PAX6 and NEUROG2 are involved in the specification of glutamatergic projection neurons, and overexpression of Neurog2 in mouse embryonic stem cells and cultured mouse cortical 
astroglia instructs the reprogramming of functional glutamatergic neurons (Berninger et al., 2007;

Heinrich et al., 2010; Schuurmans and Guillemot, 2002; Thoma et al., 2012). Conversely, combination

of NEUROG2 expression with specific small molecules converts human fibroblasts to cholinergic neurons (Liu et al., 2013; Smith et al., 2016). Additional studies will be required to determine if PAX6, analogous to the master regulator ASCL1, acts as pioneer factor that can access its targets even if they are bound by nucleosomes (Wapinski et al., 2013). reprogramming and predicts novel combinations of pro-neuronal transcription factors that allow the generation of clinically relevant neuronal subtypes. Thus, Convert-seq represents an effective method to identify transcriptional codes controlling multiple cell fate conversions, speaking to its potential to become a standard strategy to unravel molecular mechanisms governing cell reprogramming.

372

\section{Acknowledgments}

374 This work was supported by a Research Grant from the Japanese Ministry of Education, Culture,

375 Sports, Science and Technology (MEXT) and a Postdoctoral Fellowship for Research in Japan by the 376 Japan Society for the Promotion of Science (JSPS). The authors wish to acknowledge RIKEN GeNAS

377 for the sequencing of the libraries.

378

\section{Author Contributions}

380 Formulation of research goals and aims: JL, JWS

381 Data curation: JL, TK, CP

382 Formal analysis: JL, TK, RN, JWS

383 Funding acquisition: JL, PC, JWS

384 Performing experiments: JL, TK, RN, TC, DS, MK, FR

385 Project supervision: PC, JWS

386 Writing - original draft, review \& editing: JL, JWS

\section{Declaration of Interests}

389 All authors do not claim conflict of interest for this study. 


\section{$390 \quad$ Figure legends}

391

392

Figure 1. Generation of a heterogenous population of human induced neurons

393 (A) Overview of Convert-seq.

394 (B) Immunostaining for canonical neuronal marker genes of fibroblasts at day 0 and $\mathrm{Ci}$ and TFi at $9 \mathrm{dpi}$

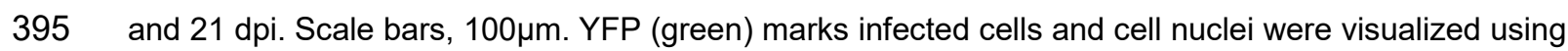

396 DAPI nuclear stain (grey).

397 (C) Quantification of immunostainings in B. $n=4$ independent experiments, error bars represent mean $398+$ SD.

399 (D) qPCR for pan-neuronal marker genes (MAP2, NRCAM, NEUN, SYN1; top), canonical neuronal 400 subtype markers (SLC17A7, GABRA1, TH, CHAT; middle) and canonical fibroblast markers (VIM, 401 SNAl1; bottom). $\mathrm{n}=3$ independent experiments, error bars represent mean + SD.

402 (E) Immunostainings for canonical neuronal subtype markers (red) of TFi at $21 \mathrm{dpi}$. Scale bars, $100 \mu \mathrm{m}$. 403 YFP (green) marks infected cells and cell nuclei were visualized using DAPI nuclear stain (grey).

404 (F) Quantification of immunostainings in E. Error bars represent mean + SD.

405

406 Figure 2. Molecular characterization of induced neurons using ScRNA-seq

407 (A) Visualization of droplet-based scRNA-seq data from Ci and TFi at 14 dpi using t-SNE $(n=3865$ 408 cells). The detected clusters are indicated by different colors.

409 (B) Heat map of the relative expression of canonical fibroblast and neuron markers along t-SNE 2.

410 (C) Heat map of the relative expression of top marker genes for each cluster in the t-SNE plot in (A).

411 (D) GO analysis of cluster-specific marker genes in clusters CL4 - CL9. Shown are top 5 GO terms 412 related to biological process (dark grey) and cellular component (light grey) for each cluster; colors as 413 in $(A)$.

414 (E) Violin plots of $\log _{2}$-transformed CPM values of marker genes in all clusters.

415 (F) Annotation of TFi clusters CL4 - CL9 based on genes differentially expressed between each cluster.

416

417 Figure 3. Convert-seq Detection of Exogenous and Endogenous Transcripts

418 (A) Schematic depicting the strategy to distinguish exogenous and endogenous sequencing reads. 
419 (B) Bulk Convert-seq on pooled and separately infected fibroblasts. Horizontal dimension; distance from 420 the 5" end of the EF1A promoter, vertical dimension; number of aligned paired-end reads. Gray arrows 421 (no overlap) and golden arrows (overlap) mark 5' and 3' junctions of exogenous ORFs.

422 (C-D) Heat maps showing $\log _{2}$-transformed count values of exogenous transcription factors after 423 alignment using Bowtie (C) and $\log _{2}$-transformed TPM values of exogenous and endogenous 424 transcription factor pairs after trimming junction sequences to $\sim 100$ base pairs and alignment using 425 Kallisto (D). For individually infected fibroblasts and Ci, 2 replicates at an $\mathrm{MOI}$ of 4 and 2 replicates at 426 an $\mathrm{MOI}$ of 8 were included. For pooled infected fibroblasts, 2 replicates at an MOI of 4 were included.

427 (E) Box plots showing increased exogenous (red) versus endogenous (blue) expression of all 428 transcription factors across all individually infected fibroblasts. Golden dots show endogenous 429 expression in samples infected with the corresponding exogenous transcription factors. Error bars 430 represent mean + SD.

431

432 Figure 4. Induced neurons resemble primary human newborn neurons in global gene expression

433 (A) Diagram of the differentiation protocol of TFi and $\mathrm{Ci}$, depicting the samples used for the time-course 434 scRNAseq experiment.

435 (B) t-SNE 2D cell maps of the time-course data. Left: cells were colored by cluster identity. Right: cells 436 were colored by sample identity. Fibroblasts ( $n=78$ cells), Ci at 9 dpi $(n=87$ cells), TFi at 9 dpi $(n=$ 437 129), Ci at $21 \mathrm{dpi}(\mathrm{n}=15$ cells), TFi at $21 \mathrm{dpi}(\mathrm{n}=137$ cells $)$.

438 (C) Heat map of the relative expression of top marker genes for each cluster in the t-SNE plot in (B).

439 (D) Lineage relationships of fibroblasts, $\mathrm{Ci}$, TFi and primary cortical and medial ganglionic eminence 440 cells $(n=4261$ cells $)$ in an adjacency network.

441 (E) Visualization of relative expression values of exogenous transcription factors on $t$-SNE plots.

443 Figure 5. Association of developmental trajectories with exogenous expression profiles.

444 (A) Pseudo-temporal ordering of cells (Fluidigm C1) based on the expression of 3,322 developmental 445 genes $(n=446$ cells). Small inset shows the same plot colored by pseudo-temporal values.

446 (B) Branch-specific relative expression of neurogenic genes (top row), cell cycle-related genes (middle 447 row) and genes related to alternative developmental fates (down row) along pseudo-time. 
448 (C) GO analysis of genes differentially expressed in branch 1 (top panel) and branch 2 (bottom panel).

449 (D) Identification of exogenous transcription factors with branch-specific enrichment based on Fisher's 450 exact tests.

451 (E) Top panels: Quantification of TUBB3+ and MAP2+ cells in fibroblasts infected with the complete 452 TF-pool (gray), branch 2-enriched transcription factors (orange) and unenriched transcription factors 453 (purple) at 9 dpi and 21 dpi. $n=5$ independent experiments, error bars represent mean + SD. Bottom 454 panels: Representative images of immunostainings for MAP2 (red) at $21 \mathrm{dpi}$; colors as in top panels. 455 YFP (green) marks infected cells and cell nuclei were visualized using DAPI nuclear stain (grey). Scale 456 bars, $100 \mu \mathrm{m}$.

457 (F-I) Neuronal differentiation, loss of fibroblast characteristics and acquisition of alternative 458 developmental fates as revealed by qPCR for pan-neuronal marker genes (MAP2, NRCAM, NEUN, 459 SYN1; F), canonical neuronal subtype markers (VGLUT1, GABA, TH, CHAT; G), canonical fibroblast 460 markers (VIM, SNAI2; H) and branch 1-enriched genes (AREG, PTHR1; I at 9 dpi and $21 \mathrm{dpi}$; colors as 461 in E. $n=4$ independent experiments, error bars represent mean + SD.

462

463 Figure 6. Identification of GRNs controlling subtype specification of induced neurons.

464 (A) Neuronal subtype-specific gene regulatory networks on the basis of significant associations 465 (Fisher's exact test, $\mathrm{p}<0.05$ ). Neuronal subtype-specific genes are colored. Exogenous transcription 466 factors with at least 3 edges to neuronal subtype-specific genes are shown in black. Exogenous 467 transcription factors with less than 3 edges are shown in gray.

468 (B) Edge-normalized network summarizing the associations of exogenous transcription factors (shown 469 in A) with neuronal subtype-specific GRNs.

470 (C) Validation of GRNs as revealed by quantifications of immunostainings for VGLUT1, CHAT, TH and 471 GABA of fibroblasts separately infected with the TF-pool (gray) or with exogenous transcription factors 472 shown in the boxes at $21 \mathrm{dpi}$ (colored). $n=5$ independent experiments, error bars represent mean + 473 SD.

474 (D-E) Left panels: visualization of Combinations Scores (CS) of glutamatergic-specific (D) and 475 cholinergic-specific (E) GRNs. Exogenous transcription factors associated with genes showing highest 476 CS in each gene regulatory network are shown in black, all other exogenous transcription factors are 477 shown in gray. Neuronal subtype-specific genes are colored. Right panels: Validation of novel 
478 combinations of exogenous transcription factors by quantification of immunostainings for VGLUT1,

479 CHAT, TH and GABA of Ci (light gray), fibroblasts infected with the complete TF-pool (dark gray) and

480 fibroblasts infected with novel combinations (color) at 21 dpi. $n=4$ independent experiments, error bars

481 represent mean + SD.

482 (F-G) The generation of the action potential in iN infected with DLX2, NEUROG2, PAX6, ZIC1 (F) or

483 DLX1, ISL1, NEUROG2, PAX6 (G). Representative traces in the presence (upper panel) or absence

484 (lower panel) of extracellular $\mathrm{Na}^{+}$were recorded using the current-clamp protocol.

485

486

487

488

489

490

491

492

493

494

495

496

497

498

499

500 


\section{STAR Methods}

\section{Key Resources Table}

\begin{tabular}{|c|c|c|}
\hline $\begin{array}{l}\text { REAGENT or } \\
\text { RESOURCE }\end{array}$ & SOURCE & IDENTIFIER \\
\hline \multicolumn{3}{|l|}{ Antibodies } \\
\hline $\begin{array}{l}\text { Mouse monoclonal anti- } \\
\text { Tubulin, beta, Class III (Tuj1) }\end{array}$ & Covance & $\begin{array}{l}\text { Cat\# MMS-435P; } \\
\text { RRID:AB_2313773 }\end{array}$ \\
\hline $\begin{array}{l}\text { Mouse monoclonal anti- } \\
\text { Map2 }\end{array}$ & Abcam & $\begin{array}{l}\text { Cat\# ab11267; } \\
\text { RRID:AB_297885 }\end{array}$ \\
\hline $\begin{array}{l}\text { Rabbit polyclonal anti- } \\
\text { Synapsin1 }\end{array}$ & Abcam & $\begin{array}{l}\text { Cat\# ab64581; } \\
\text { RRID:AB_1281135 }\end{array}$ \\
\hline Rabbit polyclonal anti-vGlut1 & Synaptic Systems & $\begin{array}{l}\text { Cat\# } 135303 \\
\text { RRID:AB_887875 }\end{array}$ \\
\hline $\begin{array}{l}\text { Mouse monoclonal anti- } \\
\text { GABA }\end{array}$ & Abcam & $\begin{array}{l}\text { Cat\# ab86186 } \\
\text { RRID:AB_1925012 }\end{array}$ \\
\hline Rabbit polyclonal anti-TH & Abcam & $\begin{array}{l}\text { Cat\# ab112 } \\
\text { RRID:AB_297840 }\end{array}$ \\
\hline Sheet polyclonal anti-CHAT & Abcam & $\begin{array}{l}\text { Cat\# ab18736 } \\
\text { RRID:AB_2244867 }\end{array}$ \\
\hline Goat anti-mouse IgG1 & Molecular Probes & $\begin{array}{l}\text { Cat\# A-21123 } \\
\text { RRID:AB_141592 }\end{array}$ \\
\hline Goat anti-mouse IgG2a & Thermo Fisher Scientific & $\begin{array}{l}\text { Cat\# A-21133 } \\
\text { RRID:AB_2535772 }\end{array}$ \\
\hline $\begin{array}{l}\text { Goat polyclonal anti-rabbit } \\
\operatorname{lgG}(\mathrm{H}+\mathrm{L})\end{array}$ & Thermo Fisher Scientific & $\begin{array}{l}\text { Cat\# R37117 } \\
\text { RRID:AB_2556545 }\end{array}$ \\
\hline $\begin{array}{l}\text { Donkey polyclonal anti- } \\
\text { sheep IgG }(\mathrm{H}+\mathrm{L})\end{array}$ & Thermo Fisher Scientific & $\begin{array}{l}\text { Cat\# A-21098 } \\
\text { RRID:AB_2535752 }\end{array}$ \\
\hline \multicolumn{3}{|c|}{ Chemicals, Peptides and Recombinant Proteins } \\
\hline Fetal Bovine Serum (FBS) & Clontech & Cat\# 631367 \\
\hline Penicilin\&Streptomycin & Thermo Fisher Scientific & Cat\# 15140122 \\
\hline Trypsin-EDTA & Sigma-Aldrich & Cat\# 25200-114 \\
\hline Polybrene & Sigma-Aldrich & Cat\# H9268 \\
\hline Poly-D-Lysine & Sigma-Aldrich & Cat\# P6407 \\
\hline $\begin{array}{l}\text { Laminin from Engelbreth- } \\
\text { Holm-Swarm murine } \\
\text { sarcoma basement } \\
\text { membrane }\end{array}$ & Sigma-Aldrich & Cat\# L2020 \\
\hline
\end{tabular}




\begin{tabular}{|c|c|c|}
\hline $\begin{array}{l}\text { B27 Supplement (50x), } \\
\text { serum free }\end{array}$ & Thermo Fisher Scientific & Cat\# 17504044 \\
\hline N2 Supplement (100x) & Thermo Fisher Scientific & Cat\# 17502048 \\
\hline $\begin{array}{l}\text { MEM Non-Essential Amino } \\
\text { Acids Solution (100x) }\end{array}$ & Thermo Fisher Scientific & Cat\# 11140050 \\
\hline Glutamax Supplement & Thermo Fisher Scientific & Cat\# 35050061 \\
\hline $\begin{array}{l}\text { Valproic Acid for } \\
\text { Biochemistry }\end{array}$ & WAKO & Cat\# 227-01071 \\
\hline L-Ascorbic Acid & Sigma-Aldrich & Cat\# A92902 \\
\hline ROCK inhibitor Y-27632 & WAKO & Cat\# 253-00513 \\
\hline $\begin{array}{l}\mathrm{N}^{6}, 2^{\prime}-\mathrm{O} \text {-Dibutyryladenosine } \\
3^{\prime}, 5^{\prime} \text {-cyclic monophosphate } \\
\text { sodium salt }\end{array}$ & Sigma-Aldrich & Cat\# D0260 \\
\hline CHIR99021 & Abcam & Cat\# ab120890 \\
\hline SB-431542 & Sigma-Aldrich & Cat\# S4317 \\
\hline LDN-193189 & REPROCELL & Cat\# 04-0074-10 \\
\hline Human Noggin & Sigma-Aldrich & Cat\# SRP4675 \\
\hline $\begin{array}{l}\text { BDNF Recombinant human } \\
\text { protein }\end{array}$ & Thermo Fisher Scientific & Cat\# 10908010 \\
\hline $\begin{array}{l}\text { NT3 Recombinant human } \\
\text { protein }\end{array}$ & R\&D Systems & Cat\# 267-N3 \\
\hline $\begin{array}{l}\text { GDNF Recombinant human } \\
\text { protein }\end{array}$ & Thermo Fisher Scientific & Cat\# PHC7044 \\
\hline Proteinase K Solution & Thermo Fisher Scientific & Cat\# AM2548 \\
\hline $\begin{array}{l}\text { FuGENE HD Transfection } \\
\text { Reagent }\end{array}$ & Promega & Cat\# E2311 \\
\hline $\begin{array}{l}\text { HBSS, calcium, magnesium, } \\
\text { no phenol red }\end{array}$ & Thermo Fisher Scientific & Cat\# 14025092 \\
\hline RNase ONE Ribonuclease & Promega & Cat\# M4261 \\
\hline Triton X-100 & Sigma-Aldrich & Cat\# T8787 \\
\hline \multicolumn{3}{|l|}{ Critical Commercial Assays } \\
\hline $\begin{array}{l}\text { Gateway LR Clonase II } \\
\text { Enzyme mix }\end{array}$ & Thermo Fisher Scientific & Cat\# 11791100 \\
\hline $\begin{array}{l}\text { PureYield Plasmid Midiprep } \\
\text { System }\end{array}$ & Promega & Cat\# A2492 \\
\hline
\end{tabular}




\begin{tabular}{|c|c|c|}
\hline RNeasy Mini Kit & Quiagen & Cat\# 74106 \\
\hline $\begin{array}{l}\text { One-Step SYBR PrimeScript } \\
\text { RT PCR Kit II }\end{array}$ & Takara & Cat\# RR086A \\
\hline $\begin{array}{l}\text { Chromium }^{\mathrm{TM}} \text { Single Cell 3' } \\
\text { Reagent kits V1 }\end{array}$ & 10x Genomics & Cat\# CG00026 \\
\hline $\begin{array}{l}\text { KAPA Library Quantification } \\
\text { kit }\end{array}$ & KAPA BIOSYSTEMS & Cat\# 07960166001 \\
\hline ERCC RNA Spike-In Mix1 & Thermo Fisher Scientific & Cat\# 4456740 \\
\hline SMARTer Ultra Low RNA Kit & Clontech & Cat\# 634848 \\
\hline $\begin{array}{l}\text { Quant-iTTM PicoGreen } \\
\text { dsDNA Assay kit }\end{array}$ & Thermo Fisher Scientific & Cat\# P7589 \\
\hline $\begin{array}{l}\text { Nextera XT DNA Library } \\
\text { Preparation kit }\end{array}$ & Illumina & Cat\# FC-131-1024 \\
\hline Nextera XT Index Kit v2 & Illumina & Cat\# FC-131-1002 \\
\hline \multicolumn{3}{|l|}{ Deposited Data } \\
\hline Raw and analyzed data & This Paper & GEO: GSE117075 \\
\hline \multicolumn{3}{|c|}{ Experimental Models: Cell Lines } \\
\hline $\begin{array}{l}\text { Human neonatal dermal } \\
\text { fibroblasts }\end{array}$ & Lonza & Cat\# C-2509 \\
\hline HEK293T & ATCC & Cat\# CRL-3216 \\
\hline \multicolumn{3}{|l|}{ Recombinant DNA } \\
\hline $\begin{array}{l}\text { CSII-EF-RfA-IRES2- } \\
\text { VENUS-ASCL1 }\end{array}$ & This paper & $\mathrm{N} / \mathrm{A}$ \\
\hline $\begin{array}{l}\text { CSII-EF-RfA-IRES2- } \\
\text { VENUS-DLX1 }\end{array}$ & This paper & $\mathrm{N} / \mathrm{A}$ \\
\hline $\begin{array}{l}\text { CSII-EF-RfA-IRES2- } \\
\text { VENUS-DLX2 }\end{array}$ & This paper & N/A \\
\hline $\begin{array}{l}\text { CSII-EF-RfA-IRES2- } \\
\text { VENUS-FEV }\end{array}$ & This paper & $\mathrm{N} / \mathrm{A}$ \\
\hline $\begin{array}{l}\text { CSII-EF-RfA-IRES2- } \\
\text { VENUS-FOXA2 }\end{array}$ & This paper & N/A \\
\hline $\begin{array}{l}\text { CSII-EF-RfA-IRES2- } \\
\text { VENUS-FOXP2 }\end{array}$ & This paper & N/A \\
\hline $\begin{array}{l}\text { CSII-EF-RfA-IRES2- } \\
\text { VENUS-ISL1 }\end{array}$ & This paper & $\mathrm{N} / \mathrm{A}$ \\
\hline $\begin{array}{l}\text { CSII-EF-RfA-IRES2- } \\
\text { VENUS-LHX2 }\end{array}$ & This paper & N/A \\
\hline
\end{tabular}




\begin{tabular}{|c|c|c|}
\hline $\begin{array}{l}\text { CSII-EF-RfA-IRES2- } \\
\text { VENUS-NEUROD1 }\end{array}$ & This paper & $\mathrm{N} / \mathrm{A}$ \\
\hline $\begin{array}{l}\text { CSII-EF-RfA-IRES2- } \\
\text { VENUS-NEUROG2 }\end{array}$ & This paper & $\mathrm{N} / \mathrm{A}$ \\
\hline $\begin{array}{l}\text { CSII-EF-RfA-IRES2- } \\
\text { VENUS-NR2F1 }\end{array}$ & This paper & N/A \\
\hline $\begin{array}{l}\text { CSII-EF-RfA-IRES2- } \\
\text { VENUS-NR2F2 }\end{array}$ & This paper & $\mathrm{N} / \mathrm{A}$ \\
\hline $\begin{array}{l}\text { CSII-EF-RfA-IRES2- } \\
\text { VENUS-NR4A2 }\end{array}$ & This paper & $\mathrm{N} / \mathrm{A}$ \\
\hline $\begin{array}{l}\text { CSII-EF-RfA-IRES2- } \\
\text { VENUS-OLIG2 }\end{array}$ & This paper & $\mathrm{N} / \mathrm{A}$ \\
\hline $\begin{array}{l}\text { CSII-EF-RfA-IRES2- } \\
\text { VENUS-OTX2 }\end{array}$ & This paper & N/A \\
\hline $\begin{array}{l}\text { CSII-EF-RfA-IRES2- } \\
\text { VENUS-PAX6 }\end{array}$ & This paper & $\mathrm{N} / \mathrm{A}$ \\
\hline $\begin{array}{l}\text { CSII-EF-RfA-IRES2- } \\
\text { VENUS-PITX3 }\end{array}$ & This paper & $\mathrm{N} / \mathrm{A}$ \\
\hline $\begin{array}{l}\text { CSII-EF-RfA-IRES2- } \\
\text { VENUS-POU3F2 }\end{array}$ & This paper & $\mathrm{N} / \mathrm{A}$ \\
\hline $\begin{array}{l}\text { CSII-EF-RfA-IRES2- } \\
\text { VENUS-TLX3 }\end{array}$ & This paper & $\mathrm{N} / \mathrm{A}$ \\
\hline $\begin{array}{l}\text { CSII-EF-RfA-IRES2- } \\
\text { VENUS-ZIC1 }\end{array}$ & This paper & $\mathrm{N} / \mathrm{A}$ \\
\hline \multicolumn{3}{|c|}{ Software and Algorithms } \\
\hline Bowtie v1.2.2 & (Langmead et al., 2009) & $\begin{array}{l}\text { http://bowtie- } \\
\text { bio.sourceforge.net/index.shtml }\end{array}$ \\
\hline Kallisto v0.42.4 & (Bray et al., 2016) & https://pachterlab.github.io/kallisto/ \\
\hline $\mathrm{R} \vee 3.5 .2$ & $\mathrm{R}$ project & https://www.r-project.org/ \\
\hline edgeR v3.24.3 & (Robinson et al., 2010) & $\begin{array}{l}\text { https://bioconductor.org/packages/relea } \\
\text { se/bioc/html/edgeR.html }\end{array}$ \\
\hline rio v0.5.16 & CRAN & $\begin{array}{l}\text { https://cran.r- } \\
\text { project.org/web/packages/rio/index.htm }\end{array}$ \\
\hline Tidyverse v1.2.1 & CRAN & $\begin{array}{l}\text { https://cran.r- } \\
\text { project.org/web/packages/tidyverse/ind } \\
\text { ex.html }\end{array}$ \\
\hline Readxl v1.2.0 & CRAN & $\begin{array}{l}\text { https://cran.r- } \\
\text { project.org/web/packages/readxl/index. } \\
\text { html }\end{array}$ \\
\hline Reshape v0.8.8 & (Wickham, 2007) & $\begin{array}{l}\text { https://cran.r- } \\
\text { project.org/web/packages/reshape/inde } \\
\text { x.html }\end{array}$ \\
\hline pheatmap v1.0.12 & CRAN & $\begin{array}{l}\text { https://cran.r- } \\
\text { project.org/web/packages/pheatmap/in } \\
\text { dex.html }\end{array}$ \\
\hline
\end{tabular}




\begin{tabular}{|c|c|c|}
\hline data.table v1.12.0 & CRAN & $\begin{array}{l}\text { https://cran.r- } \\
\text { project.org/web/packages/data.table/in } \\
\text { dex.html }\end{array}$ \\
\hline Seurat v2.3.0 & (Butler et al., 2018) & $\begin{array}{l}\text { https://cran.r- } \\
\text { project.org/web/packages/Seurat/index } \\
\text { html }\end{array}$ \\
\hline Dendextend v1.9.0 & (Galili, 2015) & $\begin{array}{l}\text { https://cran.r- } \\
\text { project.org/web/packages/dendextend/ } \\
\text { citation.html }\end{array}$ \\
\hline monocle v2.2.0 & (Trapnell et al., 2014) & $\begin{array}{l}\text { https://bioconductor.org/packages/relea } \\
\text { se/bioc/html/monocle.html }\end{array}$ \\
\hline Cytoscape v3.7.1 & (Shannon et al., 2003) & https://cytoscape.org/ \\
\hline
\end{tabular}

501

\section{Cell culture and generation of iN}

503 Human neonatal dermal fibroblasts were purchased from Lonza (C-2509; passages 4-8) and cultured

504 in DMEM containing 10\% FBS/L-glutamine/10\% Penicilin\&Streptomycin at $37^{\circ} \mathrm{C} 5 \% \mathrm{CO}_{2}$ until virus 505 infection. For TFi generation, a total of $6 \mathrm{MOI}(\sim 0.3 \mathrm{MOI}$ per virus) were pooled into culture medium 506 containing $8 \mu \mathrm{gg}^{*} \mathrm{ml}^{-1}$ Polybrene (SIGMA) to increase infection efficiency. The virus pool was infected 507 and incubated overnight at $37^{\circ} \mathrm{C} 5 \% \mathrm{CO}_{2}$. On the following day, virus-containing medium was removed 508 and cells were incubated for an additional day in fresh culture medium at $37^{\circ} \mathrm{C} 5 \% \mathrm{CO}_{2}$. For iN 509 generation, infected cells were split onto Poly-d-lysine $\left(100 \mu \mathrm{g}^{*} \mathrm{ml}^{-1}\right.$; SIGMA) / Laminin $\left(50 \mu \mathrm{g}^{*} \mathrm{ml}^{-1}\right.$; 510 SIGMA)-coated culture plates and incubated overnight in culture medium at $37^{\circ} \mathrm{C} 5 \% \mathrm{CO}_{2}$. On the 511 following day, medium was changed to neuronal induction medium containing DMEM/F12 and 512 Neurobasal-A (Thermo Fisher Scientific) mixed at a 1:1 ratio, 2\% (vol/vol) B27 supplement and 0.5\% 513 N2 Supplement (GIBCO), 1x nonessential amino acids (Thermo Fisher Scientific), 1\% GlutaMAX

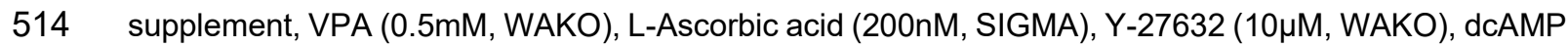
515 (0.5mM, SIGMA), 10\% FBS and 10\% Penicilin\&Streptomycin (WAKO). The concentrations of small

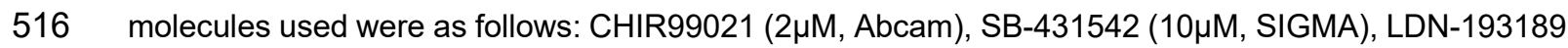
$517\left(0.5 \mu \mathrm{M}\right.$, Stemgent), Noggin (100 $\mathrm{ng}^{*} \mathrm{ml}^{-1}$, SIGMA). Neuronal induction medium was changed every third 518 day, during which the concentration of FBS was gradually reduced from $10 \%$ to $0 \%$. After 2 weeks, 519 neuronal induction medium was replaced with neuronal maturation medium without small molecules, 520 but containing $10 \mathrm{ng}^{*} \mathrm{ml}^{-1}$ BDNF (GIBCO), 10ng ${ }^{*} \mathrm{ml}^{-1} \mathrm{NT} 3$ (R\&D Systems) and 10ng ${ }^{*} \mathrm{ml}^{-1}$ GDNF (Thermo 521 Fisher Scientific) and the medium was changed every third day until further analysis.

522

523 Complementary DNA and virus generation 
524 Complementary DNA (cDNA) and viruses were generated as described previously (Shin et al., 2012).

525 Briefly, we recombined Gateway-compatible human full-length cDNA entry clones derived from RIKEN

526 BRC clone bank (http://www.brc.riken.jp/) into the pENTR lentivirus vector CSII-EF-RfA-IRES2-VENUS

527 using Gateway LR clonase II enzyme mix (Invitrogen). After Proteinase K treatment, recombined

528 plasmids were transformed into competent Escherichia coli and plasmids derived from single colonies

529 were expanded and purified using PureYield Plasmid Midiprep System (Promega). Plasmids, HIV-gp

530 and VSV envelope genes were co-transfected onto 293T cells using FuGeneHD (Roche). Supernatant-

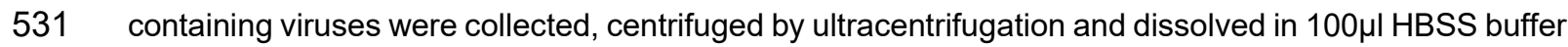

532 (WAKO) and stored at $-80^{\circ} \mathrm{C}$ for later use.

533

\section{Immunocytochemistry and quantitative RT-PCR}

535 For immunocytochemistry, cells were fixed in $4 \%$ paraformaldehyde for 20 min at room temperature 536 and permeabilized using $0.2 \%$ Triton X-100 (SIGMA) for $10 \mathrm{~min}$ at room temperature. Following 537 permeabilization, cells were pre-incubated with blocking solution ( $2 \%$ BSA, $0.2 \%$ Triton X-100) to block 538 non-specific sites for $1 \mathrm{~h}$. Primary antibodies were diluted in blocking solution and applied to cells over 539 night at $4^{\circ} \mathrm{C}$. Secondary antibodies were diluted in blocking solution and applied to cells at room 540 temperature for $1 \mathrm{~h}$. Imaging was performed using the INCell Analyzer 6000 (GE Healthcare). The 541 following primary antibodies and dilutions were used: mouse anti-TUBB3 (Covance, 1:1000), mouse 542 anti-MAP2 (Abcam, 1:500), rabbit anti-SYNAPSIN 1 (Abcam, 1:200), rabbit anti-VGLUT1 (Synaptic 543 Systems, 1:100), mouse anti-GABA (Abcam, 1:200), sheep anti-CHAT (Abcam, 1:100), rabbit anti-TH

544 (Abcam, 1:500). The following secondary antibodies and dilutions were used: goat anti-mouse IgG1 545 (GIBCO, 1:200), goat anti-mouse IgG2a (Termo Fisher Scientific, 1:200), goat anti-rabbid IgG (Thermo 546 Fisher Scientific, 1:200), donkey anti-sheep IgG (Thermo Fisher Scientific, 1:200). Human neonatal 547 dermal fibroblasts were used as negative controls. Quantification of immunostainings was performed 548 using the INCell Investigator Developer Toolbox. For quantitative RT-PCR (qRT-PCR), total RNA was 549 purified using the RNeasy Mini Kit (QIAGEN) according to the manufacturer's specification. Quality and 550 quantity of RNA was determined using a DropSense96 (Trinean). Equal amounts of RNA were reverse551 transcribed using the One-Step SYBR PrimeScript RT PCR Kit II, and cDNAs were normalized to equal 
552 amounts using primers against Gapdh. qRT-PCR was performed on a 7900HT Fast Real-Time PCR

553 system (Applied Biosystems).

554

555 Convert-seq

556 Artificial transcript model: To determine the exact nucleotide sequences flanking the ORF of each 557 exogenous transcript, we sequenced recombined plasmids using a 3730/3730xI DNA Analyzer (Applied 558 Biosystems) following the manufacturer's protocol. Briefly, we first amplified templates by PCR using a 559 primers annealing to the EF1A promoter sequence near the 5 ' end of each ORF to amplify the 5 “ junction 560 sequences and primers annealing to the IRES2 sequence near the 3' end of each ORF to amplify the 5613 3 junction sequences. After gel purification, we sequenced templates using BigDye Terminator v3.1 562 Cycle Sequencing Kits (Applied Biosystems). We integrated results derived from 3 primers (3 replicates

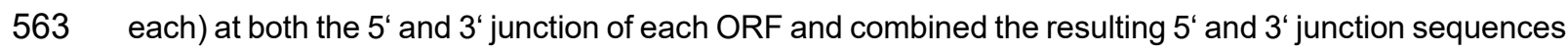
564 with known sequences of the ORFs of each transcription factor and the CSII-EF-RfA-IRES2-VENUS 565 pENTR lentivirus vector to compile the artificial exogenous transcript model. Lastly, we combined our 566 artificial exogenous transcript model with the human transcriptome (version GRCh38.p5) to obtain the 567 final artificial transcript model.

568 Read alignment with Bowtie: Reads were aligned to the artificial transcript model using Bowtie v1.2.2 569 with the default parameter settings for paired-end reads. After retrieving BED12 files using samtools

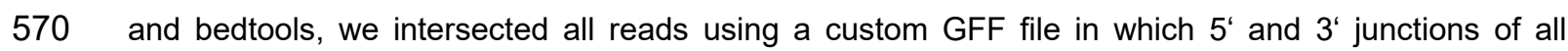
571 exogenous sequences were defined. Only reads overlapping the junction sequences by at least $5 \mathrm{bp}$ 572 were counted as specific reads. The expression values of all exogenous transcription factors were 573 quantified as count per million (CPM) and transformed to $\log _{2}(\mathrm{CPM}+1)$.

574 Read alignment with Kallisto: For alignment using Kallisto (v0.42.4), alignment to the full artificial 575 transcript model yielded many false-positive hits (Figure S3B). Therefore, we trimmed the 5' and 5763 ' junction sequences to $\sim 100$ bp on either side, which markedly reduced the number of false positive 577 hits (Figure 3D). Reads were aligned with the default parameter settings for paired-end reads. Custom 578 R scripts were used to merge transcript isoforms and compile a single expression matrix.

579 Convert-seq on Drop-seq data: Since the CellRanger Software does not provide a single FASTQ file 580 for each single-cell, we used the bcl2fastq Conversion Software to convert and demultiplex BCL files. 
581 After retrieving FASTQ files for each single cell, reads were aligned to the trimmed artificial transcript model using Kallisto with the default parameter settings in paired-end mode.

583

584 Droplet-based scRNA-seq

585 Library preparation and sequencing: Droplet-based scRNA-seq libraries were generated using the 586 Chromium $^{\mathrm{TM}}$ Single Cell 3' Reagent kits V1 (CG00026, 10x Genomics). Briefly, cell number and cell 587 viability were assessed using the Countess II Automated Cell Counter (ThermoFisher). Thereafter, cells 588 were mixed with the Single Cell Master Mix and loaded together with Single Cell 3' Gel beads and 589 Partitioning Oil into a Single Cell 3' Chip. RNA transcripts were uniquely barcoded and reverse590 transcribed in droplets. cDNAs were pooled and amplified according to the manufacturer's protocol. 591 Libraries were quantified by High Sensitivity DNA Reagents (Agilent Technologies) and the KAPA 592 Library Quantification kit (KAPA BIOSYSTEMS). Libraries then were sequenced by Illumina Hiseq 2500 593 in rapid mode.

594 Read alignment and gene quantification: Initial read alignment to hg19 human reference genome, 595 filtering and UMI counting was performed by the CellRanger Software ver 1.1.0 using default 596 parameters. This software implements STAR as an alignment tool. Data from TFi and Ci were 597 normalized to the same sequencing depth and aggregated into a single gene-barcode matrix. The 598 expression values were quantified as count per million (CPM) and transformed to $\log _{2}(C P M+1)$.

599

600 scRNAseq using the Fluidigm C1 platform

601 Library preparation and sequencing: Single cell RNA-seq analysis was performed following the 602 manufacturer's protocol (P100-7168L1, Fluidigm). Briefly, cell number and cell viability were assessed 603 using the Countess II Automated Cell Counter (ThermoFisher). After priming medium size C1 Single604 cell Open App IFCs, 250 cells/ $\mu \mathrm{L}$ were loaded and capture efficiency and cell morphology was assessed 605 using the IN Cell Analyzer 6000 (GE Healthcare). To exclude chambers loaded with no cells, more than 606 one cell (cell doublets) or dead cells for downstream analysis, we took 11 z-stacking images per 607 chamber. Next, cells were lysed with 20,000-fold diluted ERCC RNA Spike-In Mix1 (Thermo Fisher 608 Scientific) and reverse transcription (RT) and cDNA amplification were performed using the SMARTer 609 Ultra Low RNA Kit for the Fluidigm C1 $1^{\text {TM }}$ System (Clontech). The amplified cDNAs were harvested into 61096 well plates and quantified with Quant-iT TM PicoGreen dsDNA Assay kit. Library preparation was 
611 performed with the Nextera XT DNA Library Preparation kit (Illumina), Nextera XT Index Kit v2 (Illumina)

612 and AMpure XP beads (Beckman Coulter). Libraries were quantified by High Sensitivity DNA Reagents

613 (Agilent Technologies) and KAPA Library Quantification kit (KAPA BIOSYSTEMS). Each of the libraries

614 were sequenced by Illumina Hiseq 2500 in highoutput mode (100bp paired end).

615 Read alignment and gene quantification: Reads were aligned to the trimmed artificial transcript model

616 using Kallisto with the default parameter settings for paired-end reads. The expression values were

617 quantified as transcript per million (TPM) and transformed to $\log _{2}(T P M+1)$.

618

619 Fluidigm C1 reversed loading protocol (backloading) for bulk RNAseq

620 To perform bulk RNAseq of a total of 96 samples, we used the Fluidigm Script Builder ${ }^{\mathrm{TM}}$ to design a

621 reversed protocol that allows to load each sample into a separate chamber, where RT and cDNA

622 amplification is performed. After priming the chips, $25 \mathrm{ng}$ of RNA of each sample was loaded into the

623 output wells on a medium size C1 Single-cell Open App IFC and the IFC was sealed using a C1 Porous

624 Barrier Tape kit (Fluidigm). RT and cDNA amplification was performed following the manufacturer's

625 protocol (P100-7168L1). We ran the backloading script for 15 min at $4^{\circ} \mathrm{C}$ and switched to the mRNA

626 seq RT and Amp script (1772x), which harvested cDNA back into the output wells. To remove remaining

627 RNA, we added Rnase One Ribonuclease (Promega) at room temperature. To quantify the cDNA, we

628 used the Quant-iT PicoGreen dsDNA Assay kit. Library preparation was performed using the Nextera

629 XT DNA Library Preparation kit (Illumina), the Nextera XT Index Kit v2 (Illumina) and Ampure XP beads

630 (Beckman Coulter). Libraries were quantified using the High Sensitivity DNA Reagents (Agilent

631 Technologies) and the KAPA Library Quantification kit (KAPA BIOSYSTEMS). Libraries were

632 sequenced on the Illumina Hiseq 2500 platform in rapid mode (100bp paired end).

633

\section{Electrophysiology}

635 Conventional whole-cell current-clamp recordings were performed as described previously (Ichikawa

636 et al., 2012). All experiments were conducted at $25^{\circ} \mathrm{C}$. Patch pipettes with resistances ranging from

$6373-7 \mathrm{M} \Omega$ were pulled from capillary tubes using a DMZ-Universal puller (Zeitz Instruments GmbH,

638 Martinsried, Germany) and then back-filled with intracellular solution. Action potentials were recorded

639 by a patch clamp amplifier (axopatch 200B; Axon Instruments, Foster City, CA) with a series of

640 current step from 0 to $200 \mathrm{pA}$ with a 2,000-ms duration. The action potentials were monitored and 
641 stored using pCLAMP software (Molecular Devices, LLC., CA) after digitizing the analog signals at 5

$642 \mathrm{kHz}$ (DigiData 1322A; Axon Instruments, Foster City, CA). For patch-clamp recordings, the

643 extracellular solution (ECS) consisted of the following: $137 \mathrm{mM} \mathrm{NaCl}, 5 \mathrm{mM} \mathrm{KCl}, 0.44 \mathrm{mM} \mathrm{KH}_{2} \mathrm{PO}_{4}$,

$6440.33 \mathrm{mM} \mathrm{Na}_{2} \mathrm{HPO}_{4}, 10 \mathrm{mM}$ glucose, $12 \mathrm{mM} \mathrm{NaHCO}_{3}, 0.5 \mathrm{mM} \mathrm{MgCl}_{2}$, and $10 \mathrm{mM} \mathrm{HEPES}$, adjusted to

$645 \mathrm{pH} 7.4$ with tris(hydroxymethyl)aminomethane. To examine the $\mathrm{Na}^{+}$selectivity, extracellular $136 \mathrm{mM}$

$646 \mathrm{NaCl}$ was substituted with equimolar extracellular $\mathrm{LiCl}\left(\mathrm{Na}^{+}\right.$-free $\left.\mathrm{ECS}\right)$. To record ionic currents under

647 physiological conditions, intracellular solution containing $150 \mathrm{mM} \mathrm{KCl}, 10 \mathrm{mM} \mathrm{HEPES}$, and $2 \mathrm{mM}$

648 magnesium adenosine triphosphate ( $\mathrm{pH} 7.2$ by tris(hydroxymethyl)aminomethane) was used.

649

\section{Computational methods for scRNA-seq data}

651 Quality control, cell clustering and $t$-SNE visualization: All analyzes and visualization of data were

652 conducted in an R environment. Clustering and t-SNE visualization was performed using the R package

653 'Seurat' (Satija et al., 2015) (v2.3.0). For droplet-based scRNA-seq data, genes expressed in less than

6543 cells and cells expressing less than 1000 genes or more than 4500 genes were removed. In addition,

655 we removed cells expressing more than $2 \%$ mitochondrial genes, indicative of dead cells. PCA was

656 performed on the $\sim 1000$ most variable genes after regressing out the number of $\mathrm{UMI}$ and the

657 percentage of mitochondrial genes. Using the 20 most significant principal components (PCs), we

658 projected individual cells based on their PC scores onto a single two-dimensional map using t-SNE

659 (Van Der Maaten and Hinton, 2008). Gene expression heatmap along t-SNE2 was obtained by dividing

660 cells into 40 groups based on their t-SNE2 scores, averaging gene expression within each group and

661 scaling expression values by column. For Fluidigm C1 data, we excluded chambers containing no cells,

662 multiple cells or cells exhibiting morphological features of cell death based on visual inspection using

663 the IN Cell Analyzer 6000 (GE Healthcare). Additionally, cells expressing either of the two housekeeping

664 genes Actb and Gapdh (encoding $\beta$-actin and glyceraldehyde-3-phosphate dehydrogenase,

665 respectively) at less than three standard deviations below the mean were scored as unhealthy and

666 removed. After applying these filters, 78 fibroblasts, 216 cells for the time-point $9 \mathrm{dpi}(87 \mathrm{Ci}$ and 129

$667 \mathrm{TFi})$ and 152 cells for the time-point $21 \mathrm{dpi} \mathrm{(15} \mathrm{Ci} \mathrm{and} 137 \mathrm{TFi}$ ) remained, yielding 446 cells in total.

668 Genes expressed in less than 3 cells were removed. PCA was performed on the $\sim 5000$ most variable

669 genes. Using the 5 most significant principal components (PCs), we projected individual cells based on 
670 their PC scores onto a single two-dimensional map using t-SNE. Hierarchical clustering was performed

671 on cells and on PCA scores using Euclidean distance metric.

672 Differential expression test and GO analysis: Marker genes of each cluster were determined using a

673 likelyhood ratio test based on zero-inflated data $(p<1 e-4)$. We used marker genes which showed, on

674 average, at least 3-fold and 2-fold enrichment (droplet-based scRNA-seq data and Fluidigm C1 data,

675 respectively) in a cluster compared to all other clusters. GO analysis was performed using the

676 PANTHER data base (http://www.pantherdb.org/) which uses Fisher's Exact tests with FDR multiple

677 test correction.

678 Construction of cellular network: The cellular network was constructed by computing a pairwise 679 correlation matrix of all cells in our time-course data and the primary cortical and medial ganglionic 680 eminence cells (Nowakowski et al., 2017). Next, we generated a weighted adjacency network graph 681 using the perfuse force-directed layout in Cytoscape and visualized cells as nodes connected to other 682 cells via edges if the Pearson pairwise correlation between two cells was $>=0.4$.

683 Pseudotemporal ordering: Pseudotemporal ordering of cells was performed using the $\mathrm{R}$ package 684 'Monocle' (Trapnell et al., 2014) (v2.2.0). For unsupervised ordering, we used genes differentially 685 expressed between cells at day 0 (fibroblasts) and Ci and TFi at day 9 and day 21 (qval < 0.1; 10`000 686 genes). To determine genes that are significantly branch-dependent $\left(p<10^{-4}\right)$, we applied the BEAM 687 algorithm. GO analysis for branch-dependent genes was performed using genes that met the following 688 criteria: 1) $p<0.01$ in a likelyhood ratio test based on zero-inflated data; 2 ) absolute $\log _{2}$ fold changes 689 between the branch under consideration and others were larger than 2. GO analysis for genes that 690 changed significantly as a function of pseudotime was performed using genes that met the following 691 criteria: 1) $p<10^{-4}$ of differentialGeneTest; 2) among the top 1000 genes showing positive or negative 692 correlation with pseudotime values. For semi-supervised ordering, we used $\sim 3000$ genes previously 693 implicated in nervous system development (GO:0007399), circulatory system development 694 (GO:0072359), urogenital system development (GO:0001655), heart development (GO:0007507), 695 mesenchyme development (GO:0060485), ear development (GO:0043583), muscle structure 696 development (GO:0061061), stem cell development (GO:0048864), pancreas development 697 (GO:0031016) and skeletal system development (GO:0001501). GO analysis was performed using 698 genes that met the following criteria: 1) $p<0.01$ in a likelyhood ratio test based on zero-inflated data; 699 2) absolute $\log _{2}$ fold changes between the branch under consideration and others were larger than 2. 
700 To determine exogenous transcription factors that are significantly branch dependent, expression

701 values were binarized $(0=$ not expressed, $1=$ expressed $)$. Then we performed Fisher's exact tests to

702 calculate the significance of association of a given exogenous TF with each cluster. Exogenous

703 transcription factors with $p<0.05$ were considered significantly enriched.

704 GRN assembly: To construct neuronal subtype-specific GRNs, we calculated the significance of 705 association of each exogenous transcription factors with all other genes using Fisher's exact tests.

706 Exogenous TFs were attributed to neuronal subtype-specific GRNs based on the following criteria: 1) $p$

$707<0.05$ in Fisher's exact test; 2) at least 3 edges to 3 neuronal subtype-specific genes. GRNs were

708 visualized with Cytoscape using the organic layout.

709 Combination score: The CS represents the -log10-transformed p-value of the significance (Mann-

710 Whitney $U$ test) of increased gene expression in cells containing at least 2 of the predicted exogenous

711 transcription factors in a network compared to all other cells.

712

\section{Statistics}

714 Statistical analyses were performed using $\mathrm{R}$ and detailed in the corresponding figure legends. All

715 Stundent's $t$-tests are two-sided.

716

717 Code availability

718 All analysis code used in this study is available upon request. Custom code for the main analytical steps

719 will be uploaded on GitHub at the day of publication.

720

721 Data availability

722 All sequence data used in this study have been deposited in the NCBI Gene Expression Omnibus

723 database and will be accessible through accession number GSE117075 upon publication.

724

725

726

727

728

729 


\section{References}

731 Adamson, B., Norman, T.M., Jost, M., Cho, M.Y., Nunez, J.K., Chen, Y., Villalta, J.E., Gilbert, L.A.,

732 Horlbeck, M.A., Hein, M.Y., et al. (2016). A Multiplexed Single-Cell CRISPR Screening Platform

733 Enables Systematic Dissection of the Unfolded Protein Response. Cell 167, 1867-1882 e21.

734 Berninger, B., Costa, M.R., Koch, U., Schroeder, T., Sutor, B., Grothe, B., and Gotz, M. (2007).

735 Functional Properties of Neurons Derived from In Vitro Reprogrammed Postnatal Astroglia. J.

736 Neurosci. 27, 8654-8664.

737 Bray, N.L., Pimentel, H., Melsted, P., and Pachter, L. (2016). Near-optimal probabilistic RNA-seq

738 quantification. Nat Biotechnol 34, 525-527.

739 Butler, A., Hoffman, P., Smibert, P., Papalexi, E., and Satija, R. (2018). A n a ly s i s Integrating

740 single-cell transcriptomic data across different conditions, technologies, and species. 36.

741 Caiazzo, M., Dell'Anno, M.T., Dvoretskova, E., Lazarevic, D., Taverna, S., Leo, D., Sotnikova, T.D.,

742 Menegon, A., Roncaglia, P., Colciago, G., et al. (2011). Direct generation of functional dopaminergic

743 neurons from mouse and human fibroblasts. Nature 476, 224-227.

744 Chanda, S., Ang, C.E., Davila, J., Pak, C., Mall, M., Lee, Q.Y., Ahlenius, H., Jung, S.W., Sudhof, T.C.,

745 and Wernig, M. (2014). Generation of induced neuronal cells by the single reprogramming factor

746 ASCL1. Stem Cell Reports 3, 282-296.

747 Chapouton, P., Gartner, A., and Gotz, M. (1999). The role of Pax6 in restricting cell migration between

748 developing cortex and basal ganglia. Development 126, 5569-5579.

749 Chen, S., Sanjana, N.E., Zheng, K., Shalem, O., Lee, K., Shi, X., Scott, D.A., Song, J., Pan, J.Q.,

750 Weissleder, R., et al. (2015). Genome-wide CRISPR screen in a mouse model of tumor growth and

751 metastasis. Cell 160, 1246-1260.

752 Dixit, A., Parnas, O., Li, B., Chen, J., Fulco, C.P., Jerby-Arnon, L., Marjanovic, N.D., Dionne, D.,

753 Burks, T., Raychowdhury, R., et al. (2016). Perturb-Seq: Dissecting Molecular Circuits with Scalable

754 Single-Cell RNA Profiling of Pooled Genetic Screens. Cell 167, 1853-1866 e17.

755 Galili, T. (2015). Data and text mining dendextend: an R package for visualizing, adjusting and

756 comparing trees of hierarchical clustering. 31, 3718-3720.

757 Gascon, S., Masserdotti, G., Russo, G.L., and Gotz, M. (2017). Direct Neuronal Reprogramming:

758 Achievements, Hurdles, and New Roads to Success. Cell Stem Cell 21, 18-34.

759 Heinrich, C., Blum, R., Gascón, S., Masserdotti, G., Tripathi, P., Sánchez, R., Tiedt, S., Schroeder, T.,

760 Götz, M., and Berninger, B. (2010). Directing astroglia from the cerebral cortex into subtype specific

761 functional neurons. PLoS Biol. 8.

762 Hsu, P.D., Lander, E.S., and Zhang, F. (2014). Development and applications of CRISPR-Cas9 for

763 genome engineering. Cell 157, 1262-1278.

764 Hu, W., Qiu, B., Guan, W., Wang, Q., Wang, M., Li, W., Gao, L., Shen, L., Huang, Y., Xie, G., et al.

765 (2015). Direct Conversion of Normal and Alzheimer's Disease Human Fibroblasts into Neuronal Cells

766 by Small Molecules. Cell Stem Cell 17, 204-212.

767 Jaitin, D.A., Weiner, A., Yofe, I., Lara-Astiaso, D., Keren-Shaul, H., David, E., Salame, T.M., Tanay,

768 A., van Oudenaarden, A., and Amit, I. (2016). Dissecting Immune Circuits by Linking CRISPR-Pooled

769 Screens with Single-Cell RNA-Seq. Cell 167, 1883-1896 e15. 
Jeff, G., Schnapp, B.J., and Sheetz, M.P. (1988). @ 1988 Nature Publishing Group. Nature 331, 450. Jiang, H., Xu, Z., Zhong, P., Ren, Y., Liang, G., Schilling, H.A., Hu, Z., Zhang, Y., Wang, X., Chen, S., et al. (2015). Cell cycle and p53 gate the direct conversion of human fibroblasts to dopaminergic neurons. Nat. Commun. 6, 1-14.

Kallur, T., Gisler, R., Lindvall, O., and Kokaia, Z. (2008). Pax6 promotes neurogenesis in human neural stem cells. Mol Cell Neurosci 38, 616-628.

Kim, J., Su, S.C., Wang, H., Cheng, A.W., Cassady, J.P., Lodato, M.A., Lengner, C.J., Chung, C.Y., Dawlaty, M.M., Tsai, L.H., et al. (2011). Functional integration of dopaminergic neurons directly converted from mouse fibroblasts. Cell Stem Cell 9, 413-419.

Langmead, B., Trapnell, C., Pop, M., and Salzberg, S.L. (2009). Ultrafast and memory-efficient alignment of short DNA sequences to the human genome. Genome Biol 10, R25.

Li, X., Zuo, X., Jing, J., Ma, Y., Wang, J., Liu, D., Zhu, J., Du, X., Xiong, L., Du, Y., et al. (2015). Small-Molecule-Driven Direct Reprogramming of Mouse Fibroblasts into Functional Neurons. Cell Stem Cell 17, 195-203.

Li, X.J., Du, Z.W., Zarnowska, E.D., Pankratz, M., Hansen, L.O., Pearce, R.A., and Zhang, S.C. (2005). Specification of motoneurons from human embryonic stem cells. Nat Biotechnol 23, 215-221. Liu, M.L., Zang, T., Zou, Y., Chang, J.C., Gibson, J.R., Huber, K.M., and Zhang, C.L. (2013). Small molecules enable neurogenin 2 to efficiently convert human fibroblasts into cholinergic neurons. Nat Commun 4, 2183.

Liu, M.L., Zang, T., and Zhang, C.L. (2016). Direct Lineage Reprogramming Reveals Disease-Specific Phenotypes of Motor Neurons from Human ALS Patients. Cell Rep. 14, 115-128.

Liu, X., Li, F., Stubblefield, E.A., Blanchard, B., Richards, T.L., Larson, G.A., He, Y., Huang, Q., Tan, A.C., Zhang, D., et al. (2012). Direct reprogramming of human fibroblasts into dopaminergic neuronlike cells. Cell Res. 22, 321-332.

Liu, Y., Yu, C., Daley, T.P., Wong, W.H., Wernig, M., and Qi, L.S. (2018). Resource CRISPR Activation Screens Systematically Identify Factors that Drive Neuronal Fate and Resource CRISPR Activation Screens Systematically Identify Factors that Drive Neuronal Fate and Reprogramming. Stem Cell 23, 758-771.e8.

Van Der Maaten, L.J.P., and Hinton, G.E. (2008). Visualizing high-dimensional data using t-sne. J. Mach. Learn. Res. 9, 2579-2605.

Masserdotti, G., Gascon, S., and Gotz, M. (2016). Direct neuronal reprogramming: learning from and for development. Development 143, 2494-2510.

Mazzoni, E.O., Mahony, S., Closser, M., Morrison, C.A., Nedelec, S., Williams, D.J., An, D., Gifford, D.K., and Wichterle, H. (2013). Synergistic binding of transcription factors to cell-specific enhancers programs motor neuron identity. Nat. Neurosci. 16, 1219-1227.

Mertens, J., Paquola, A.C.M., Ku, M., Hatch, E., Böhnke, L., Ladjevardi, S., McGrath, S., Campbell, B., Lee, H., Herdy, J.R., et al. (2015). Directly Reprogrammed Human Neurons Retain AgingAssociated Transcriptomic Signatures and Reveal Age-Related Nucleocytoplasmic Defects. Cell Stem Cell 17, 705-718.

Nowakowski, T.J., Bhaduri, A., Pollen, A.A., Alvarado, B., Mostajo-Radji, M.A., Di Lullo, E., Haeussler, 
810 M., Sandoval-Espinosa, C., Liu, S.J., Velmeshev, D., et al. (2017). Spatiotemporal gene expression 811 trajectories reveal developmental hierarchies of the human cortex. Science (80-. ). 358, 1318-1323.

812 Pang, Z.P., Yang, N., Vierbuchen, T., Ostermeier, A., Fuentes, D.R., Yang, T.Q., Citri, A., Sebastiano,

813 V., Marro, S., Sudhof, T.C., et al. (2011). Induction of human neuronal cells by defined transcription 814 factors. Nature 476, 220-223.

815 Pfisterer, U., Kirkeby, A., Torper, O., Wood, J., Nelander, J., Dufour, A., Bjorklund, A., Lindvall, O., 816 Jakobsson, J., and Parmar, M. (2011). Direct conversion of human fibroblasts to dopaminergic 817 neurons. Proc. Natl. Acad. Sci. 108, 10343-10348.

818 Picelli, S., Faridani, O.R., Bjorklund, A.K., Winberg, G., Sagasser, S., and Sandberg, R. (2014). Full819 length RNA-seq from single cells using Smart-seq2. Nat Protoc 9, 171-181.

820 Rackham, O.J., Firas, J., Fang, H., Oates, M.E., Holmes, M.L., Knaupp, A.S., Consortium, F., Suzuki, 821 H., Nefzger, C.M., Daub, C.O., et al. (2016). A predictive computational framework for direct

822 reprogramming between human cell types. Nat Genet 48, 331-335.

823 Ramskold, D., Luo, S., Wang, Y.C., Li, R., Deng, Q., Faridani, O.R., Daniels, G.A., Khrebtukova, I., 824 Loring, J.F., Laurent, L.C., et al. (2012). Full-length mRNA-Seq from single-cell levels of RNA and 825 individual circulating tumor cells. Nat Biotechnol 30, 777-782.

826 Robinson, M.D., Mccarthy, D.J., and Smyth, G.K. (2010). edgeR : a Bioconductor package for 827 differential expression analysis of digital gene expression data. 26, 139-140.

828 Ryoji Amamoto, and Arlotta, P. (2014). Development-Inspired Reprogramming of the Mammalian 829 Central Nervous System. Science (80-. ). 343.

830 Satija, R., Farrell, J.A., Gennert, D., Schier, A.F., and Regev, A. (2015). Spatial reconstruction of 831 single-cell gene expression data. Nat. Biotechnol. 33, 495-502.

832 Schuurmans, C., and Guillemot, F. (2002). Molecular mechanisms underlying cell fate specification in 833 the developing telencephalon. Curr. Opin. Neurobiol. 12, 26-34.

834 Shalem, O., Sanjana, N.E., and Zhang, F. (2015). High-throughput functional genomics using

835 CRISPR-Cas9. Nat. Rev. Genet. 16, 299-311.

836 Shannon, P., Markiel, A., Ozier, O., Baliga, N.S., Wang, J.T., Ramage, D., Amin, N., Schwikowski, B., 837 and Ideker, T. (2003). Cytoscape: a software environment for integrated models of biomolecular 838 interaction networks. Genome Res 13, 2498-2504.

839 Shin, J.W., Suzuki, T., Ninomiya, N., Kishima, M., Hasegawa, Y., Kubosaki, A., Yabukami, H.,

840 Hayashizaki, Y., and Suzuki, H. (2012). Establishment of single-cell screening system for the rapid

841 identification of transcriptional modulators involved in direct cell reprogramming. Nucleic Acids Res

$84240, \mathrm{e} 165$.

843 Smith, D.K., Yang, J., Liu, M.L., and Zhang, C.L. (2016). Small Molecules Modulate Chromatin

844 Accessibility to Promote NEUROG2-Mediated Fibroblast-to-Neuron Reprogramming. Stem Cell

845 Reports 7, 955-969.

846 Son, E.Y., Ichida, J.K., Wainger, B.J., Toma, J.S., Rafuse, V.F., Woolf, C.J., and Eggan, K. (2011).

847 Conversion of mouse and human fibroblasts into functional spinal motor neurons. Cell Stem Cell 9, $848 \quad 205-218$.

849 Stoykova, A., Treichel, D., Hallonet, M., and Gruss, P. (2000). Pax6 modulates the dorsoventral 
patterning of the mammalian telencephalon. J Neurosci 20, 8042-8050. (2012). Ectopic expression of neurogenin 2 alone is sufficient to induce differentiation of embryonic stem cells into mature neurons. PLoS One 7.

Trapnell, C., Cacchiarelli, D., Grimsby, J., Pokharel, P., Li, S., Morse, M., Lennon, N.J., Livak, K.J.,

855 Mikkelsen, T.S., and Rinn, J.L. (2014). The dynamics and regulators of cell fate decisions are revealed by pseudotemporal ordering of single cells. Nat Biotechnol 32, 381-386.

857 Treutlein, B., Lee, Q.Y., Camp, J.G., Mall, M., Koh, W., Shariati, S.A., Sim, S., Neff, N.F., Skotheim,

858 J.M., Wernig, M., et al. (2016). Dissecting direct reprogramming from fibroblast to neuron using single-

859 cell RNA-seq. Nature 534, 391-395.

860 Tsunemoto, R., Lee, S., Szücs, A., Chubukov, P., Sokolova, I., Blanchard, J.W., Eade, K.T.,

861 Bruggemann, J., Wu, C., Torkamani, A., et al. (2018). Diverse reprogramming codes for neuronal

862 identity. Nature.

863 Victor, M.B., Richner, M., Hermanstyne, T.O., Ransdell, J.L., Sobieski, C., Deng, P.Y., Klyachko, V.A., 864 Nerbonne, J.M., and Yoo, A.S. (2014). Generation of human striatal neurons by microRNA-dependent 865 direct conversion of fibroblasts. Neuron 84, 311-323.

866 Vierbuchen, T., and Wernig, M. (2011). Direct lineage conversions: unnatural but useful? Nat

867 Biotechnol 29, 892-907.

868 Vierbuchen, T., Ostermeier, A., Pang, Z.P., Kokubu, Y., Südhof, T.C., and Wernig, M. (2010). Direct conversion of fibroblasts to functional neurons by defined factors. Nature 463, 1035-1041.

870 Wapinski, O.L., Vierbuchen, T., Qu, K., Lee, Q.Y., Chanda, S., Fuentes, D.R., Giresi, P.G., Ng, Y.H., 871 Marro, S., Neff, N.F., et al. (2013). XHierarchical mechanisms for direct reprogramming of fibroblasts 872 to neurons. Cell 155, 621-635.

873 Wickham, H. (2007). Reshaping Data with the reshape Package. 21.

874 Xu, Z., Jiang, H., Zhong, P., Yan, Z., Chen, S., and Feng, J. (2016). Direct conversion of human

875 fibroblasts to induced serotonergic neurons. Mol. Psychiatry 21, 62-70.

876 Xue, Y., Ouyang, K., Huang, J., Zhou, Y., Ouyang, H., Li, H., Wang, G., Wu, Q., Wei, C., Bi, Y., et al.

877 (2013). Direct conversion of fibroblasts to neurons by reprogramming PTB-regulated microRNA

878 circuits. Cell 152, 82-96.

879 Yan, Y., Yang, D., Zarnowska, E.D., Du, Z., Werbel, B., Valliere, C., Pearce, R.A., Thomson, J.A., and

880 Zhang, S.C. (2005). Directed differentiation of dopaminergic neuronal subtypes from human

881 embryonic stem cells. Stem Cells 23, 781-790.

882 Yun, K., Potter, S., and Rubenstein, J.L. (2001). Gsh2 and Pax6 play complementary roles in

883 dorsoventral patterning of the mammalian telencephalon. Development 128, 193-205.

884 Zhang, X., Huang, C.T., Chen, J., Pankratz, M.T., Xi, J., Li, J., Yang, Y., Lavaute, T.M., Li, X.J., Ayala, 885 M., et al. (2010). Pax6 is a human neuroectoderm cell fate determinant. Cell Stem Cell 7, 90-100.

886 Zheng, G.X., Terry, J.M., Belgrader, P., Ryvkin, P., Bent, Z.W., Wilson, R., Ziraldo, S.B., Wheeler,

887 T.D., McDermott, G.P., Zhu, J., et al. (2017). Massively parallel digital transcriptional profiling of single 888 cells. Nat Commun 8, 14049. 
FIGURE 1

A

Part 1: Virus infection

Pooled transcription

factor library

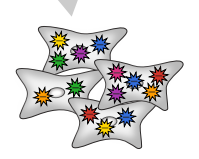

Part 2: Reprogramming

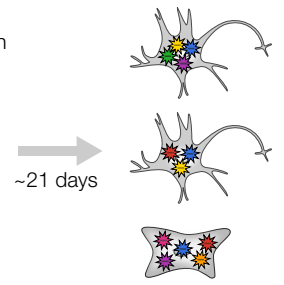

Part 3: Single-cell RNA-seq

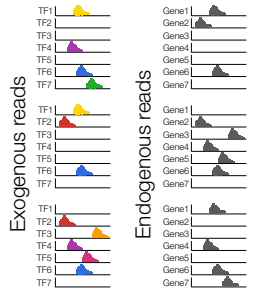

Part 4: GRN assembly
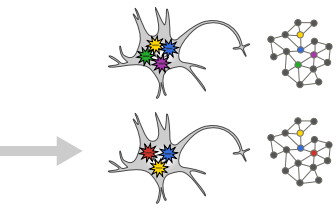

清罚
Part 5: Validations

Dopaminergic

neurons

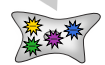

Cholinergic

( neurons

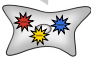

B

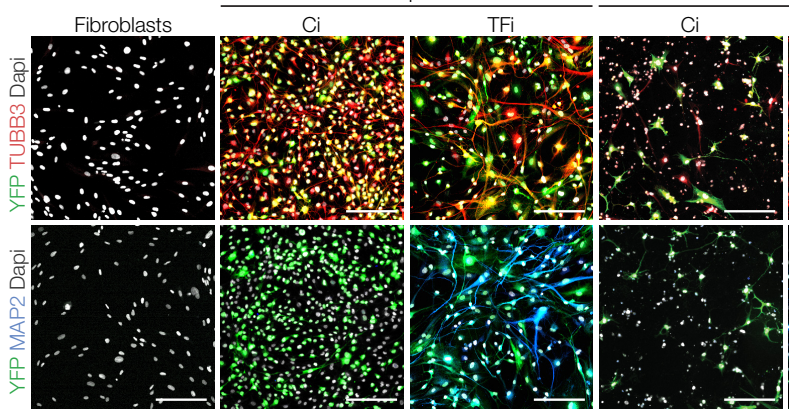

$\mathrm{Ci}=$ chemically induced $/ \mathrm{TFi}=$ transcription factor-induced

D
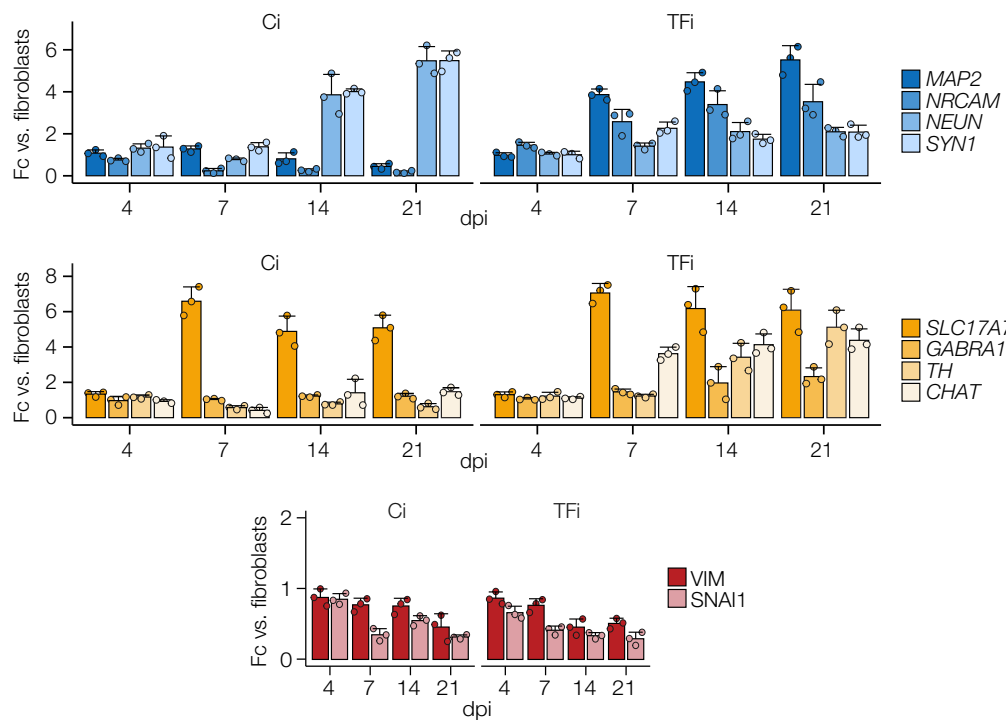

C

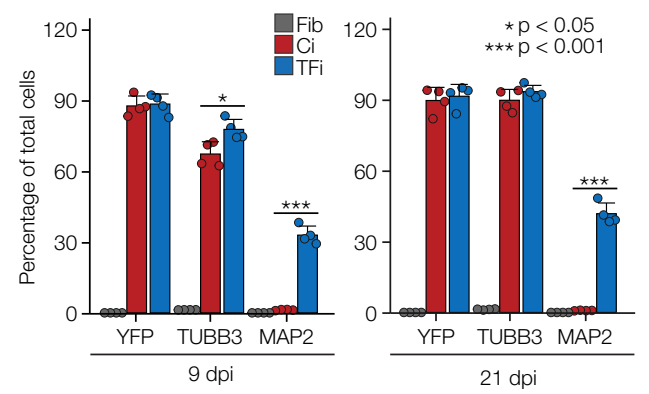

E

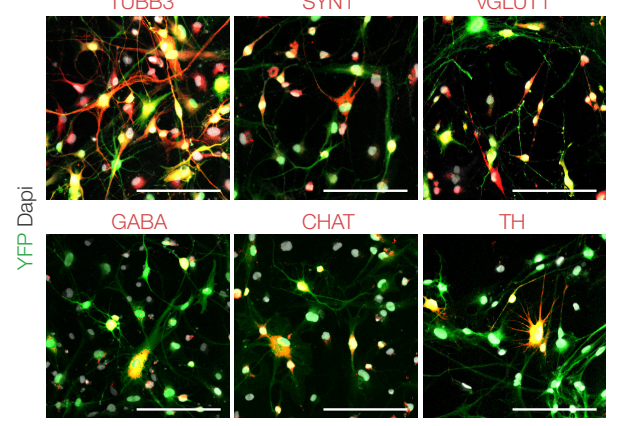

F

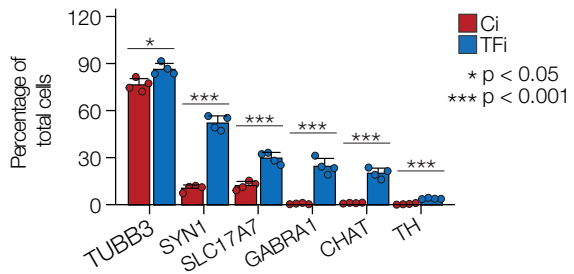


A

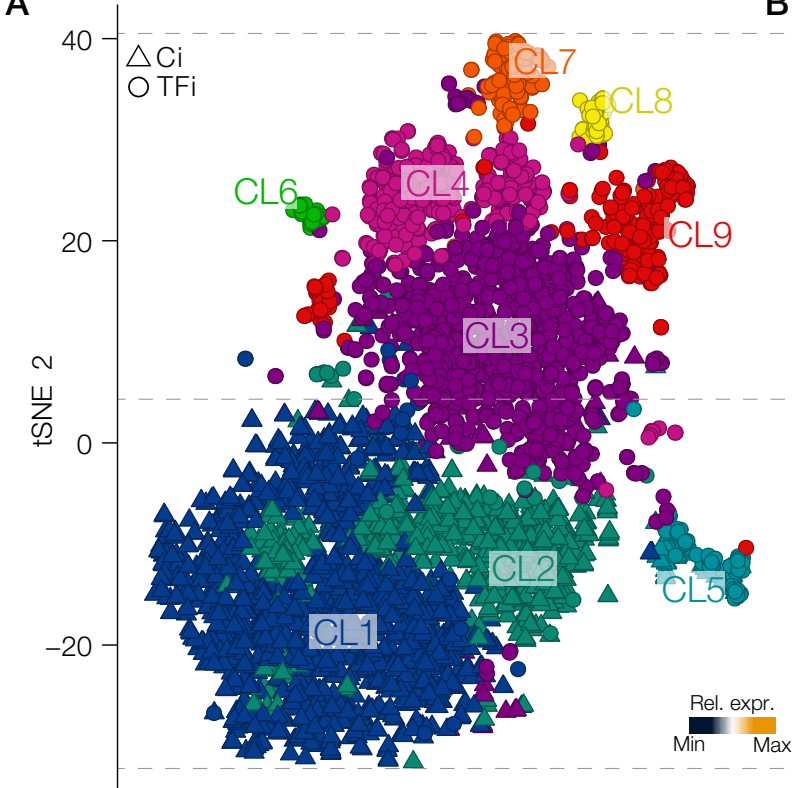

C

$$
-20
$$

Rel. expr.

Min Max
B

Neuronal genes Fibroblast genes

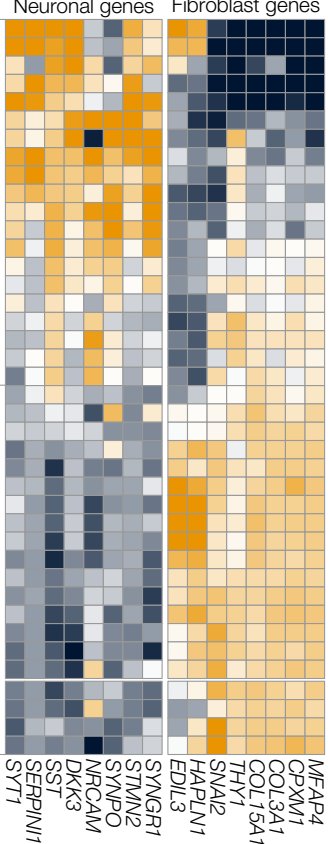

tSNE 1
20

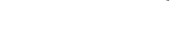

\section{D}

cell communication cellular response to chemical stimulus regulatiesponse to organic substance synapse part extracellular region neuron part plasma membrane

02468 $-\log _{10}(P$ value $)$

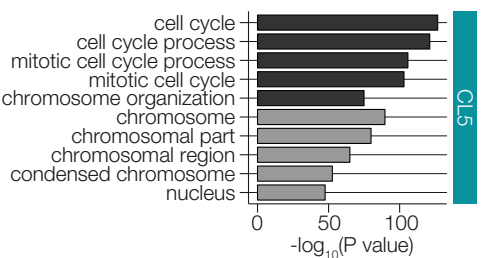

regulation of signaling regulation of response to stimulus cell communication multicellular organism development plasma membrane part plasma membrane cell periphery synaptic membrane \begin{tabular}{lllll}
\hline 0 & 2 & 4 & 1 & 1 \\
0 & 4 & 6 & 8
\end{tabular} $-\log _{10}(\mathrm{P}$ value $)$

$$
\begin{array}{r}
\text { localization } \\
\text { anatomical structure morphogenesis } \\
\text { locomotion } \\
\text { cell motility } \\
\text { cell communication } \\
\text { receptor complex } \\
\text { filopodium } \\
\text { neuron part }
\end{array}
$$

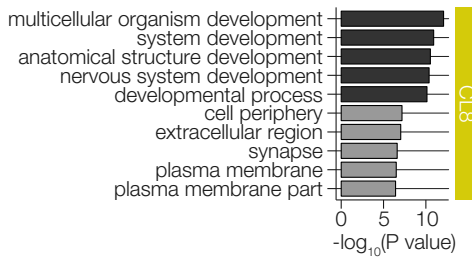
nervous system development
cell morphogenesis
neuron projection development
neuron development intrinsic component of membran cell periphery plasma membrane

integral component of membrane
extracellular region

integral component of membrane
extracellular region

$\square$ Biological process

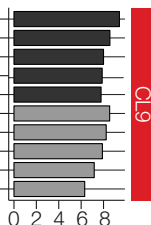
$-\log _{10}(P$ value $)$
$F$
E

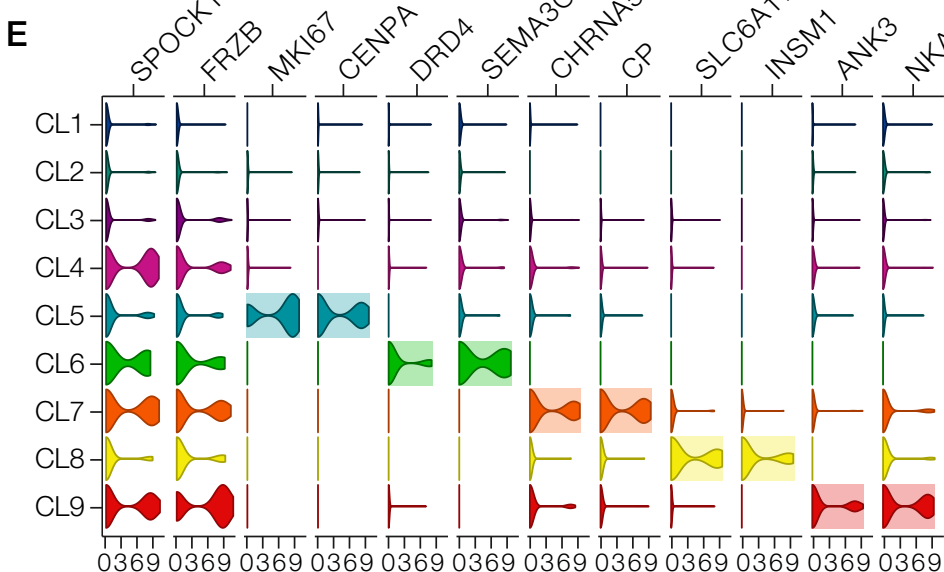

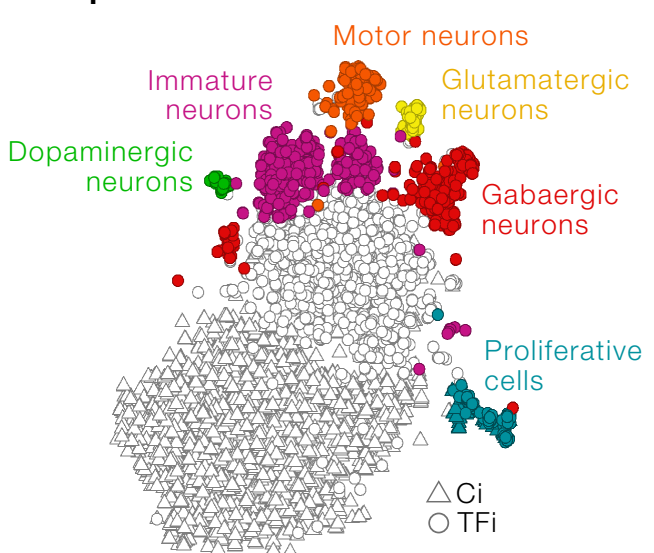




\section{FIGURE 4}

A

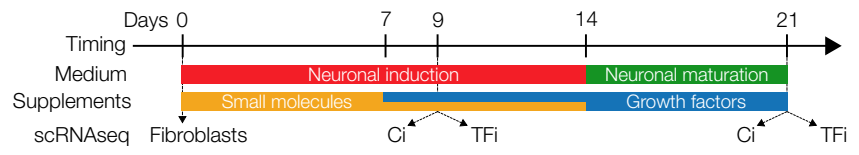

B

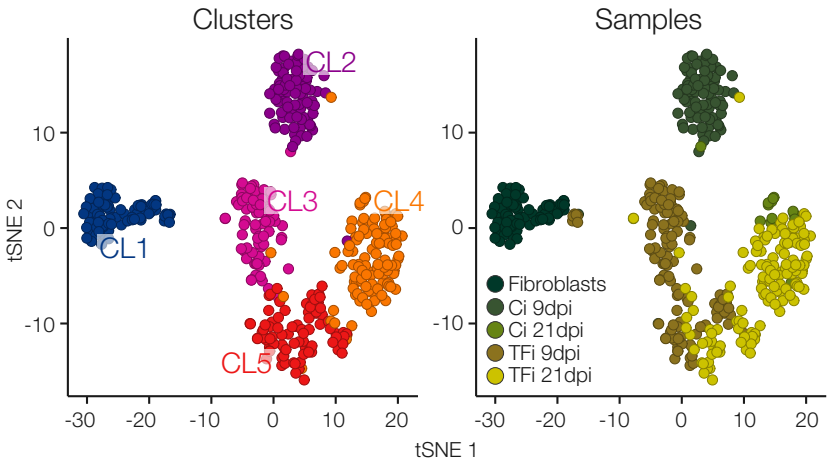

C

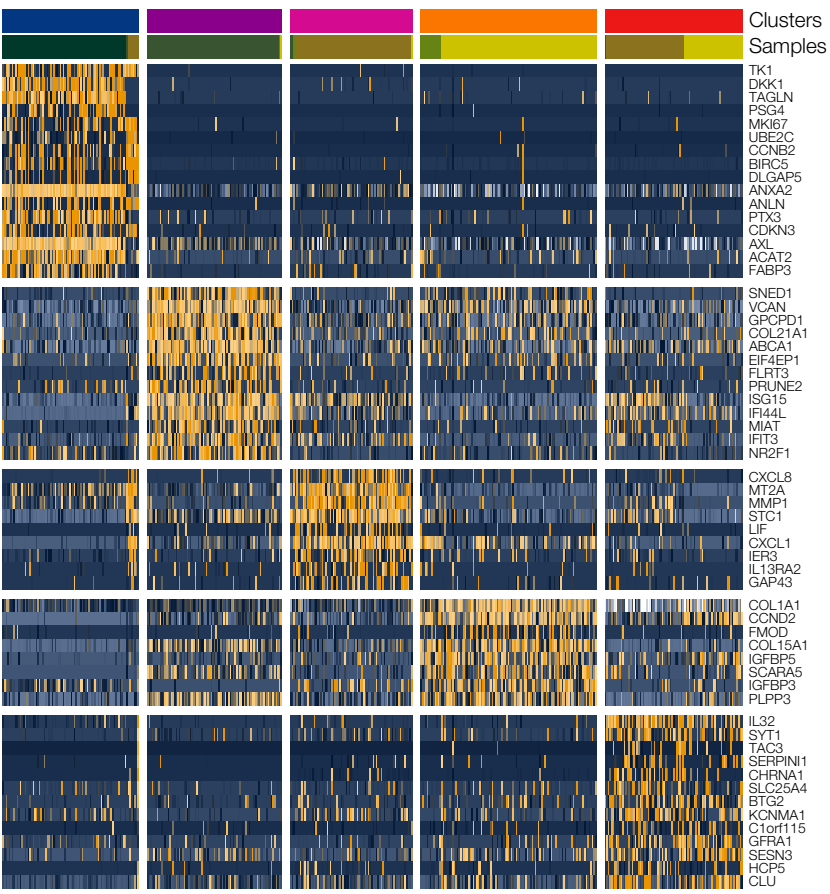

D

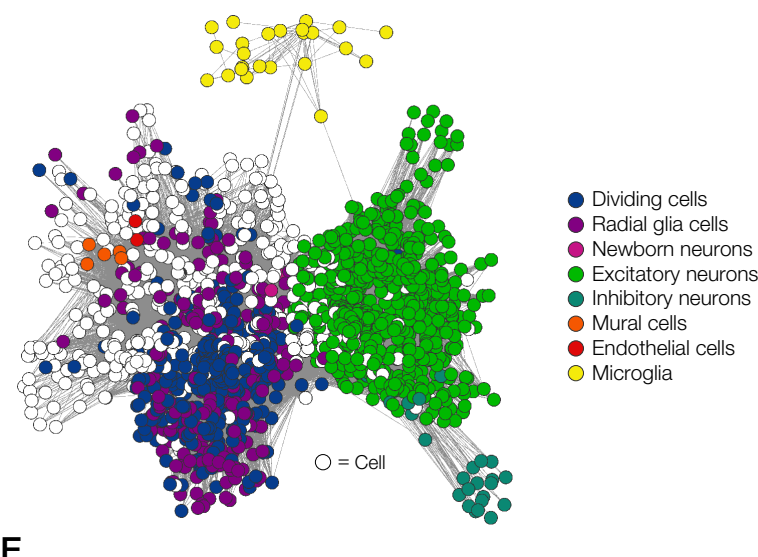

E
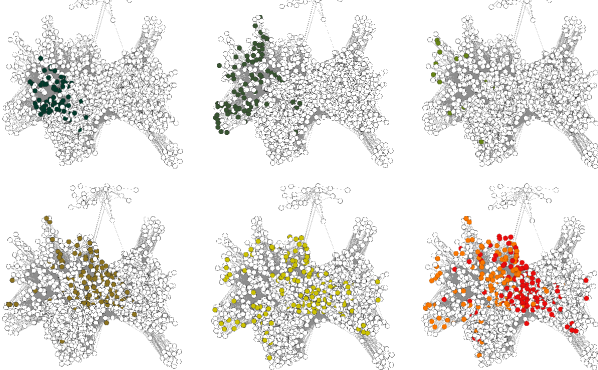

- Fibroblasts - Ci 9dpi Ci 21dpi TFi $9 d p i$
OTFi $21 d p i$

F

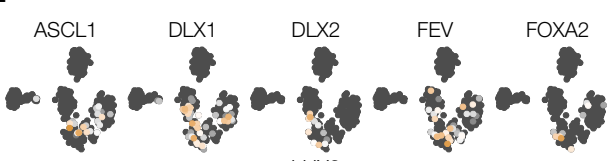

FOXP2 ISL1 LHX2 NEUROD1 NEUROG2

NR2F1 NR2F2 NR4A2

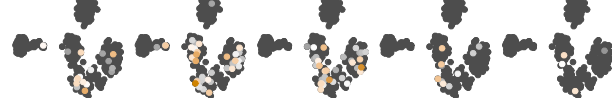

PAX POU
- CL4

- CL5 


\section{FIGURE 5}

A

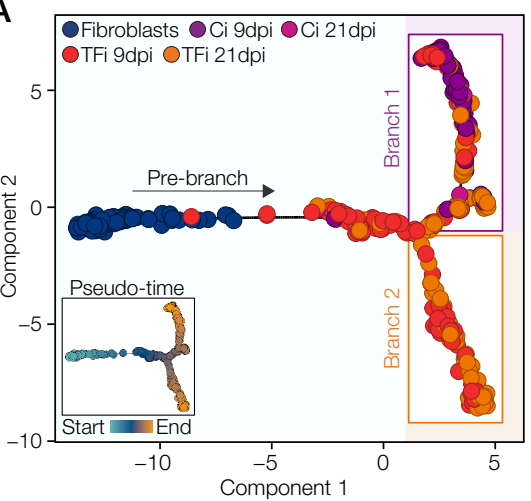

D
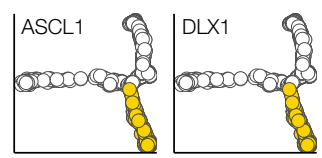

FOXP2 8


8
8

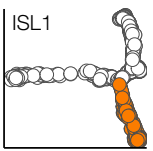

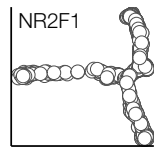

$\begin{array}{ll}\text { PAX6 } & 8 \\ 020000058 & 8\end{array}$

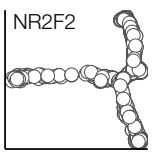

PITX3 8
000098

8
8

$$
\text { Component } 1
$$
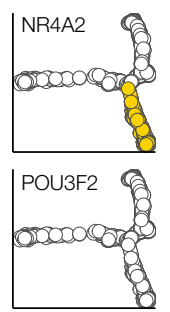$$
\text { Component } 1
$$

F

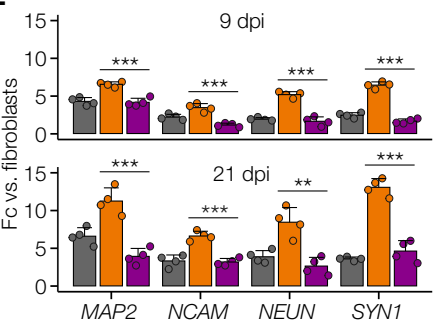

G

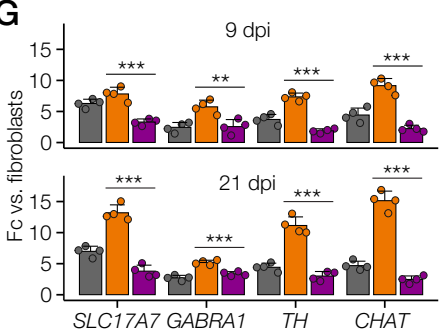

B
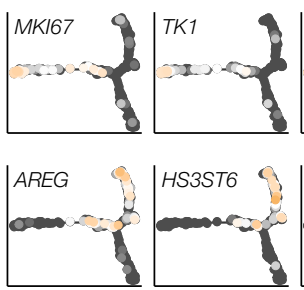

Min

Max
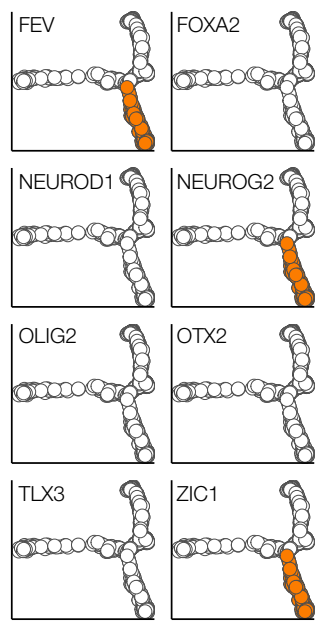

$0.05>p>1 e^{-3}$

$1 e^{-3}>p>1 e^{-5}$

$1 e^{-5}>p$
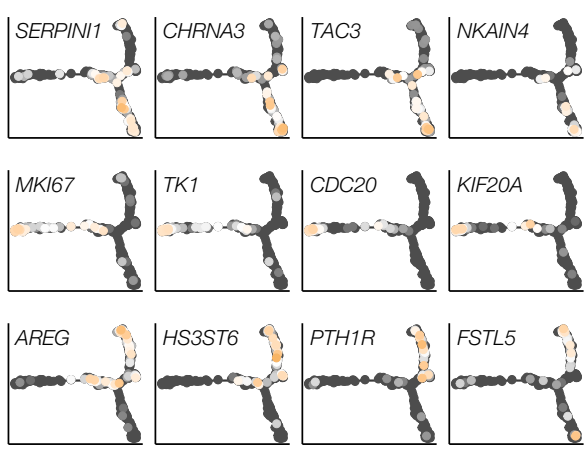

Relative expression

\section{E}
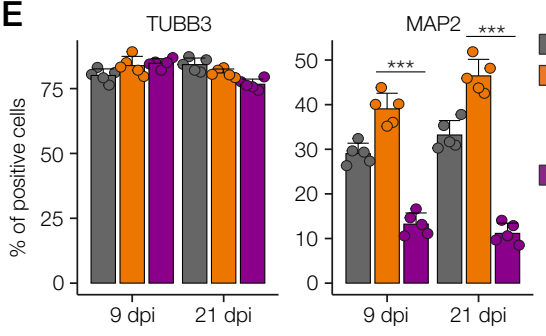

$\square$ TF-POOL

ASCL1, DLX1, DLX2, FEV, FOXP2, ISL1, NEUROG2, NR4A2, PAX6, ZIC1

FOXA2, LHX2, NEUROD1, NR2F1, NR2F2, OLIG2, OTX2, PITX2, POU3F2, TLX2 *** $p<0.001$
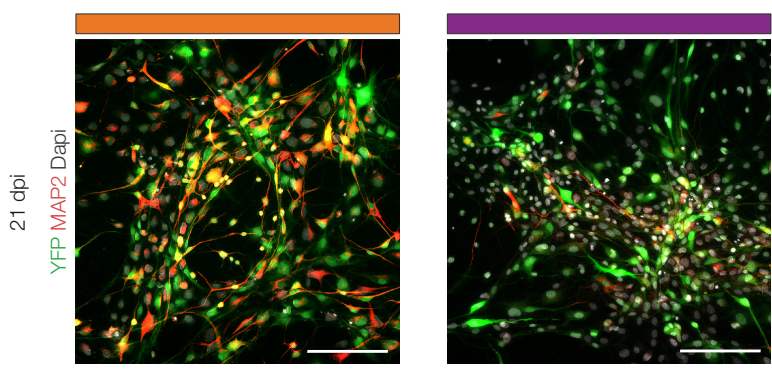

\section{H}
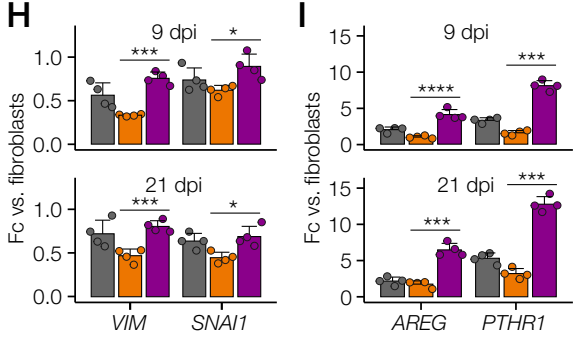

$\star p<0.05$

$\star \star p<0.01$ $\star \star \star \mathrm{p}<0.001$ 
A
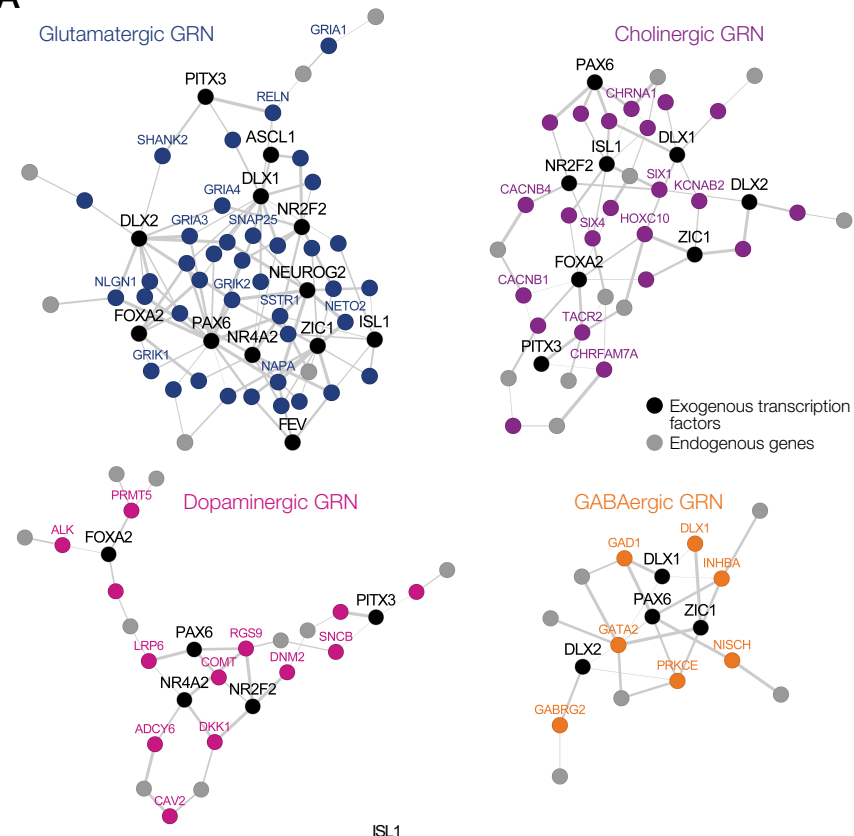

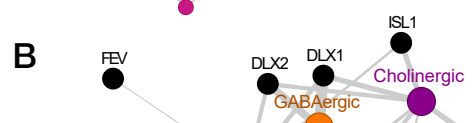

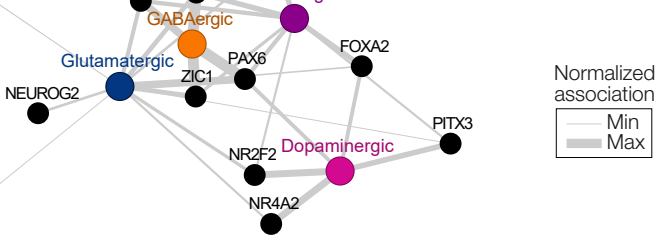

D

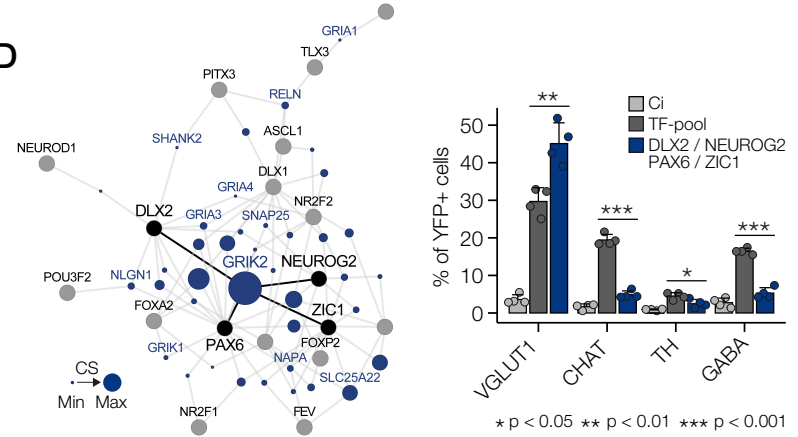

F
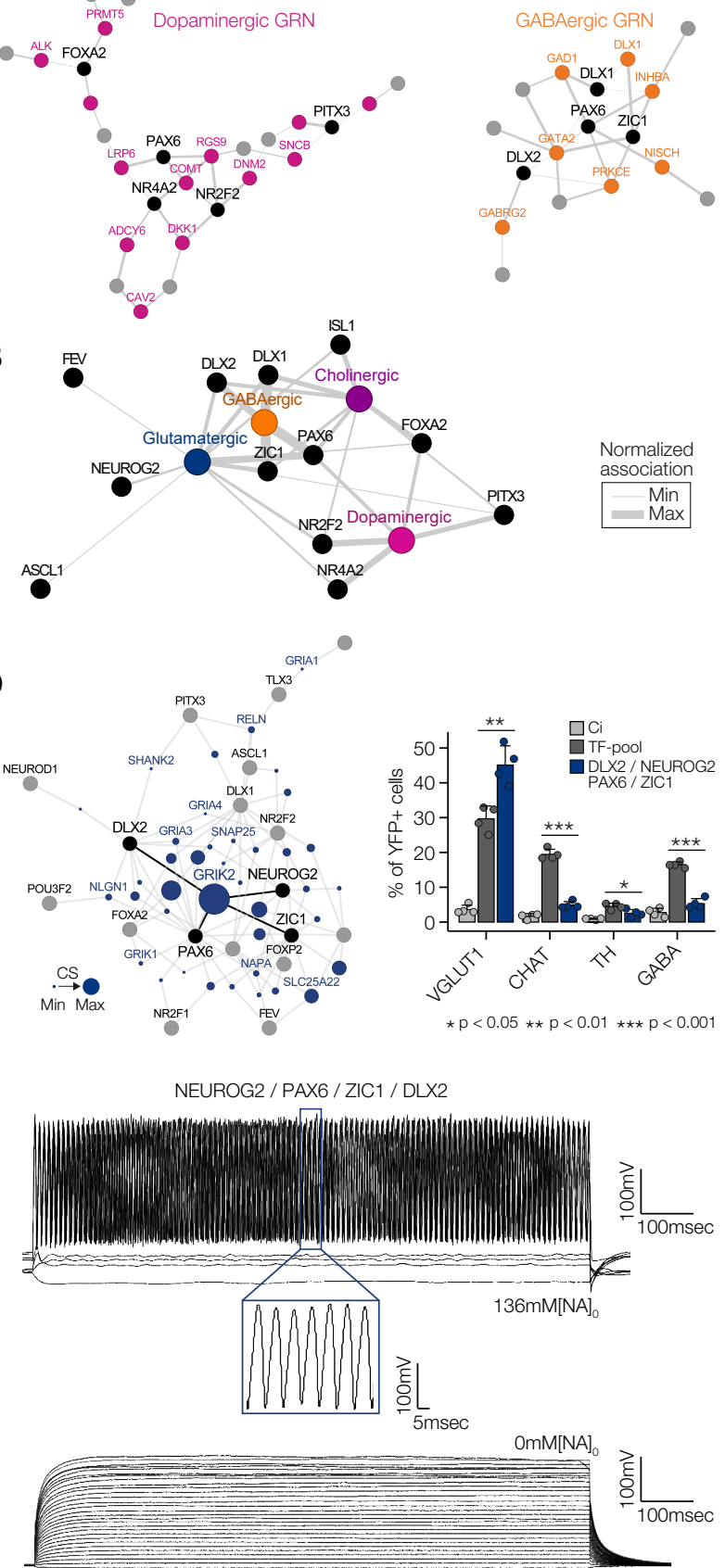

C

ASCL1 DLX1 DLX2 FEV FOXA2 ISL1 NEUROG2 NR2F2 NR4A2 PAX6

FOXA2 NR2F2 NR4A2 PAX6 PITX3

DLX1 DLX2 PAX6 ZIC1

\section{E}
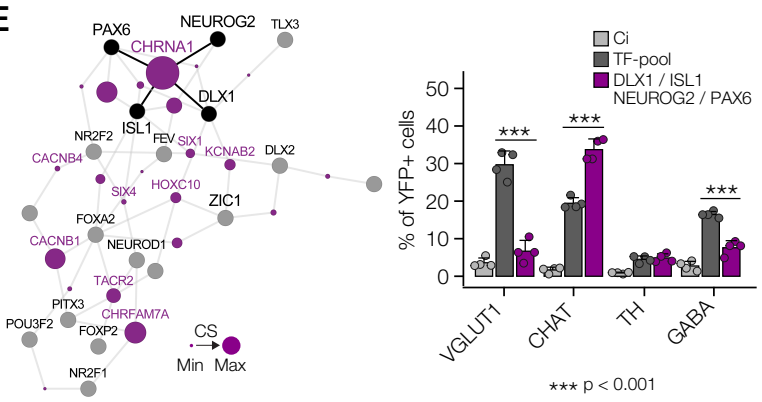

G

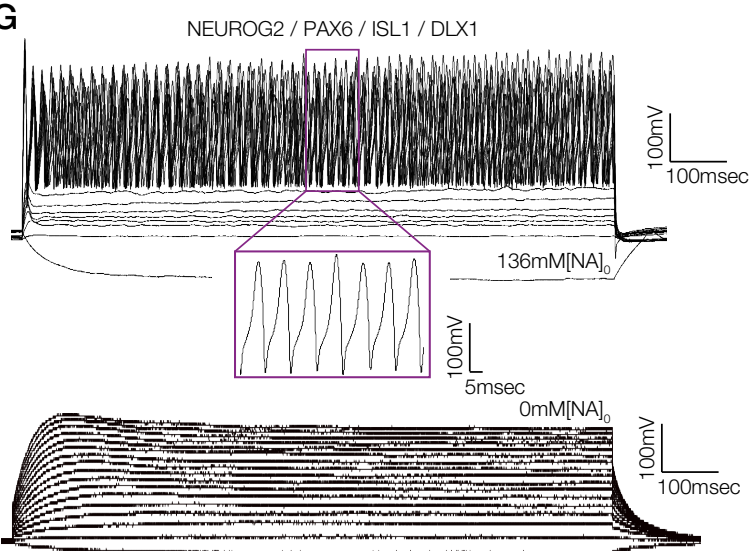

